\title{
Evidence of very long meandering features in the logarithmic region of turbulent boundary layers
}

\author{
N. HUTCHINS AND IVAN MARUSIC \\ Walter Bassett Aerodynamics Laboratory, Mechanical and Manufacturing Engineering, University of \\ Melbourne, Victoria 3010, Australia
}

(Received 1 February 2006 and in revised form 14 August 2006)

A regime of very long meandering positive and negative streamwise velocity fluctuations, that we term 'superstructures', are found to exist in the log and lower wake regions of turbulent boundary layers. Measurements are made with a spanwise rake of 10 hot-wires in two separate facilities (spanning more than a decade of $R e_{\tau}$ ) and are compared with existing PIV and DNS results. In all cases, we note evidence of a largescale stripiness in the streamwise velocity fluctuations. The length of these regions can commonly exceed $20 \delta$. Similar length scales have been previously reported for pipes and DNS channel flows. It is suggested that the true length of these features is masked from single-point statistics (such as autocorrelations and spectra) by a spanwise meandering tendency. Support for this conjecture is offered through the study of a synthetic flow composed only of sinusoidally meandering elongated low- and high-speed regions. From detailed maps of one-dimensional spectra, it is found that the contribution to the streamwise turbulence intensities associated with the superstructures appears to be increasingly significant with Reynolds number, and scales with outer length variables $(\delta)$. Importantly, the superstructure maintains a presence or footprint in the near-wall region, seeming to modulate or influence the near-wall cycle. This input of low-wavenumber outer-scaled energy into the near-wall region is consistent with the rise in near-wall streamwise intensities, when scaled with inner variables, that has been noted to occur with increasing Reynolds number. In an attempt to investigate these structures at very high Reynolds numbers, we also report on recent large-scale sonic anemometer rake measurements, made in the neutrally stable atmospheric surface layer. Preliminary results indicate that the superstructure is present in the log region of this atmospheric flow at $R e_{\tau}=6.6 \times 10^{5}$, and has a size consistent with outer scaling.

\section{Introduction}

Prior to the advent of particle image velocimetry (PIV) and direct numerical simulation (DNS) data, the precise form of the dominant log-region structure was largely unclear, although statistics based on fluctuating streamwise velocity $(u)$ signals at these heights (particularly the peak in the premultiplied energy spectra $k_{x} \Phi_{u u}$ and the long tails in the autocorrelations) had long hinted at the existence of highly elongated regions of uniform streamwise momentum. Elegant, yet laborious, spatio-temporal two-point correlations (performed using a static and traversing hot-wire probe) revealed the time-averaged scale and form of these structures (Kovasznay, Kibens \& Blackwelder 1970; Nakagawa \& Nezu 1981; McLean 1990; Wark, Naguib \& Robinson 1991). However, the first instantaneous snapshots of these structures were provided experimentally by PIV. Measurements in the streamwise/spanwise plane reveal that 
the log region appears to be characterized by its own streaky structure, notably at a much larger scale than the well-documented near-wall streaks (Kline et al. 1967). Tomkins \& Adrian (2003) and Ganapathisubramani et al. (2003) show instantaneous PIV examples of the $u$ stripiness in the log region. Long regions of streamwise momentum deficit are found, with high-speed fluid seeming to fill the separation between neighbouring motions. Further investigations have suggested that these long modes of uniform momentum deficit are associated with packets of hairpin vortices (Adrian, Meinhart \& Tomkins 2000; Tomkins \& Adrian 2003; Ganapathisubramani, Longmire $\&$ Marusic 2003). It was found that the elongated low-speed regions are flanked by vortical motions (believed to be the necks of hairpin structures) and that together these features are dominant contributors to the overall Reynolds shear stress at this height (Ganapathisubramani et al. 2003; Marusic \& Hutchins 2006). The low-speed regions are of the order $0.3-0.5 \delta$ wide, and typically have a length that exceeds the streamwise extent of the PIV frame (usually limited to $\sim 2 \delta$ ). Instantaneous examples of these features are given in $\S 3$ (figure 1 ). Here $\delta$ is the boundary layer thickness, and the axis system $x, y$ and $z$ refer to the streamwise, spanwise and wall-normal directions, with $u, v$ and $w$ describing the respective fluctuating velocity components.

There is some evidence in the literature that these features can attain very large streamwise dimensions in pipes, channel flows and atmospheric surface layers. From hot-film measurements in pipe flows, Kim \& Adrian (1999) and Guala, Hommema \& Adrian (2006) found that streamwise energetic modes can extend up to 12-14 pipe radii, and refer to these as very large-scale motions (VLSM). Kim \& Adrian (1999) suggest that the VLSM may be agglomerations of hairpin packets (themselves agglomerations of individual hairpin vortices). More recent analysis of large numerical domain DNS results (in particular two-dimensional spectra) have shown that in the $\log$ region, $\Phi_{u u}$ energy can reside in very long streamwise modes for larger $k_{y}$ bands (certainly $>20 h$, where $h$ is the channel half-height - see del Álamo \& Jiménez 2003; Jiménez 1998). Jiménez \& del Álamo (2004) attribute the very long $u$ fluctuations in the log region to 'passive wakes' formed downstream of smaller attached clusters of vortices. Similar models were suggested by Kovasznay and coworkers (Kovasznay 1970; Kovasznay et al. 1970; Blackwelder \& Kovasznay 1972). Large streak-like features have also been noted in the atmospheric surface layer, both in experiments and LES studies (Drobinski et al. 2004; Young et al. 2002) and have been referred to by Phillips (2003) as atmospheric Langmuir circulations (ALC), implying a formation mechanism similar to roll-cells observed in the oceans and other bodies of water.

In light of these suggested length scales, and in recognition of the limited fields of view afforded by PIV, we here employ a spanwise rake of 10 hot-wire sensors in an attempt to capture the true extent of the largest scales. The idea is to use the fluctuating signals from the rake to reconstruct the instantaneous spanwise profile of the $u$ velocity fluctuation. By projecting this signal in time and using Taylor's hypothesis (frozen convection) a view of the long high- and low-speed streaks can be constructed that covers a much larger streamwise domain than that attainable from PIV.

\section{Experimental set-up}

The hot-wire rake consists of 10 Dantec 55P16 single sensor hot-wire probes with a spanwise spacing $\left(s_{y}\right)$ of approximately $0.115 \delta$, such that the entire rake measures a spanwise domain just greater than one boundary layer thickness. The probes have $1.25 \mathrm{~mm}$ long platinum-plated tungsten wire sensing elements of $5 \mu \mathrm{m}$ diameter and are operated in constant temperature mode using an AA Lab Systems AN-1003 


\begin{tabular}{rcccccccccccc}
\hline & \multicolumn{1}{c}{$\delta$} & $\begin{array}{c}U_{\tau} \\
R e_{\tau}\end{array}$ & Facility & $\begin{array}{c}U_{\infty} \\
(\mathrm{m})\end{array}$ & $\begin{array}{c}\left.\mathrm{m} \mathrm{s}^{-1}\right) \\
\left(\mathrm{m} \mathrm{s}^{-1}\right)\end{array}$ & $l^{+}$ & \multicolumn{1}{c}{$s_{y} / \delta$} & $T^{+}$ & 0.05 & 0.10 & 0.15 & 0.50 \\
1120 & Minnesota & 0.069 & 0.24 & 6.0 & 17 & 0.114 & 0.4 & - & - & $\checkmark$ & $\checkmark$ \\
7610 & Melbourne & 0.336 & 0.34 & 9.82 & 23 & 0.118 & $(0.030)$ & 0.8 & $\checkmark$ & $\checkmark$ & $\checkmark$ & $\checkmark$ \\
14380 & Melbourne & 0.328 & 0.67 & 20.4 & 45 & 0.122 & $(0.030)$ & 3.0 & $\checkmark$ & $\checkmark$ & $\checkmark$ & $\checkmark$ \\
19960 & Melbourne & 0.321 & 0.96 & 29.8 & 63 & 0.125 & $(0.031)$ & 6.1 & $\checkmark$ & $\checkmark$ & $\checkmark$ & $\checkmark$
\end{tabular}

TABLE 1. Experimental parameters for hot-wire rake experiments.

$\begin{array}{rcccccc}R e_{\tau} & \text { Facility } & \begin{array}{c}\delta \\ (\mathrm{m})\end{array} & \begin{array}{c}U_{\tau} \\ \left(\mathrm{m} \mathrm{s}^{-1}\right)\end{array} & \begin{array}{c}U_{\infty} \\ \left(\mathrm{m} \mathrm{s}^{-1}\right)\end{array} & l^{+} & T^{+} \\ 1010 & \text { Minnesota } & 0.070 & 0.22 & 5.2 & 14 & 0.1 \\ 1910 & \text { Minnesota } & 0.064 & 0.47 & 12.1 & 30 & 0.7 \\ 2630 & \text { Minnesota } & 0.066 & 0.62 & 16.9 & 40 & 0.6 \\ 4110 & \text { Minnesota } & 0.061 & 1.06 & 29.6 & 67 & 0.8 \\ 7300 & \text { Melbourne } & 0.326 & 0.33 & 9.8 & 22 & 0.4\end{array}$

TABLE 2. Experimental parameters for single-wire spectra traverse experiments.

with overheat ratio set to 1.8. Details of the experimental conditions are given in table 1 . The boundary layer thickness $(\delta)$ was determined from a Coles law of the wall/wake fit to mean velocity profiles. The friction velocity $\left(U_{\tau}\right)$ is obtained from a Clauser chart fit (with log-law constants $\kappa=0.41$ and $A=5.0$ ); $U_{\infty}$ is the freestream velocity; $l^{+}\left(=l U_{\tau} / \nu\right)$ and $T^{+}\left(=t U_{\tau}^{2} / \nu\right)$ are the inner-scaled wire length and sample interval respectively. Measurements were made at several wall-normal stations through the log and wake regions. The low Reynolds number measurements were made in an open return suction-type boundary layer wind tunnel of working section $4.7 \times 1.2 \times 0.3 \mathrm{~m}$ (the PIV measurements reported here were made in the same facility: see Hutchins, Hambleton \& Marusic $2005 b$ for full details). The higher Reynolds number measurements (from $R e_{\tau}=7000$ to 20000 , where $R e_{\tau}=\delta U_{\tau} / v$ ) were performed in a separate facility at the University of Melbourne (open return blower-type wind tunnel with working section $27 \times 2 \times 1 \mathrm{~m}$ : see Hafez et al. 2004 for full details). The Melbourne experiments were also repeated with a smaller spanwise spacing $\left(s_{y} / \delta \approx 0.03\right)$.

In addition to the rake experiments, detailed boundary layer traverses were performed using a single probe across a range of Reynolds numbers in the two facilities. Details of these measurements are given in table 2. Streamwise velocity fluctuations were acquired at logarithmically spaced stations in $z$, with sufficient sampling frequency to resolve the smallest scales and sufficiently long sample lengths to converge the energy contained in the very largest scales. These data were used to produce the maps of one-dimensional spectra discussed in $\S 6$.

\section{PIV results}

Figure 1 shows sample $u$ fluctuations from various recent PIV measurements made at the University of Minnesota. Plots $(a, b$ and $c)$ are from combined-plane measurements, whereby stereoscopic views of the streamwise/spanwise and streamwise/ wall-normal plane are simultaneously acquired (see Hambleton, Hutchins \& Marusic 2006 for details). Plot $(d)$ is taken from $45^{\circ}$ inclined cross-stream measurements (Hutchins et al. 2005b). In all cases the shading shows negative streamwise velocity 

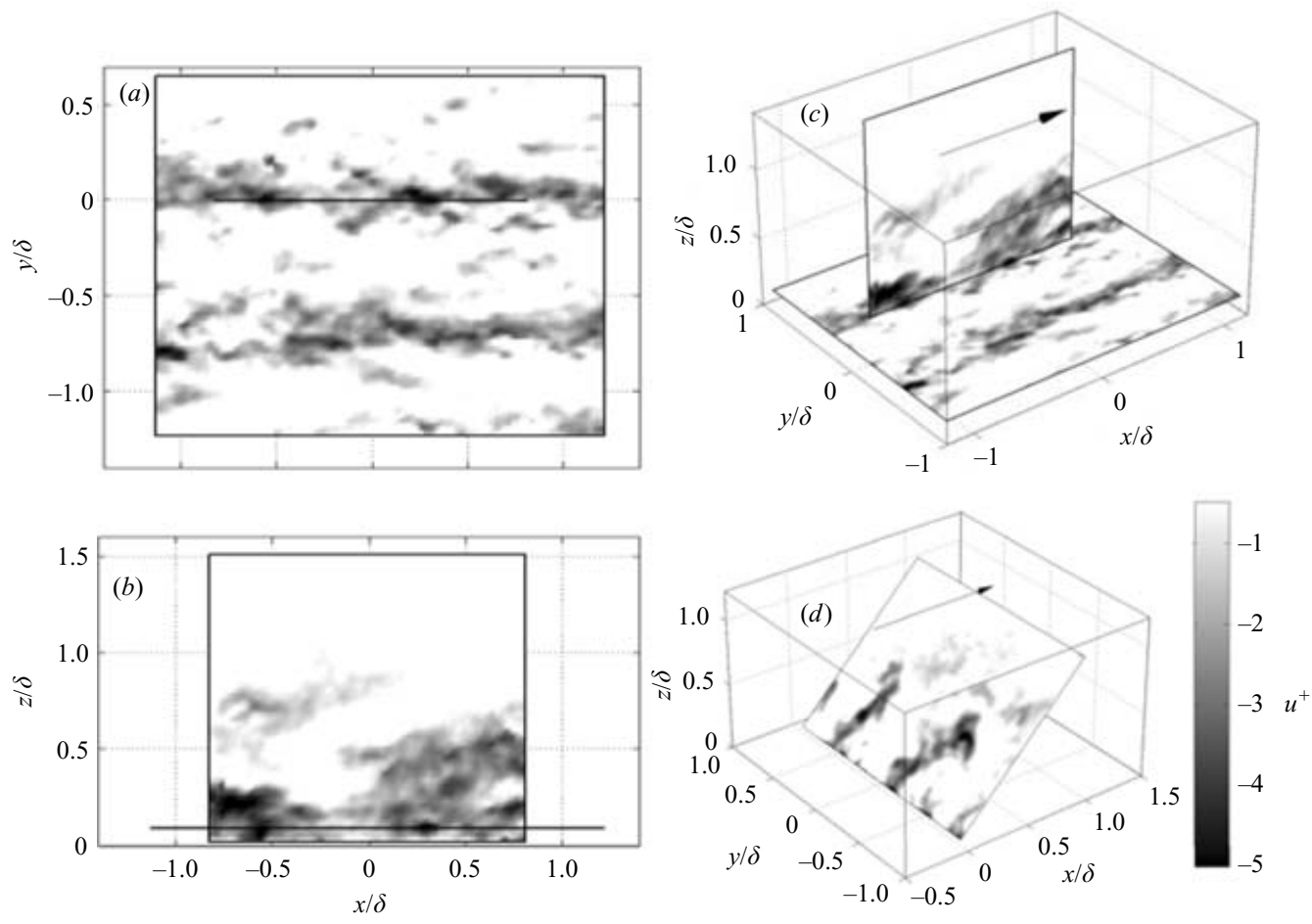

FIGURE 1. Example of instantaneous negative $u$ fluctuations from the combined-plane PIV at $R e_{\tau} \approx 1100$. (a) Plan view showing horizontal plane; $(b)$ side view showing vertical plane; (c) orthogonal projection showing both planes; $(d) 45^{\circ}$ inclined plane measurement at $R e_{\tau} \approx 1010$. Thick horizontal lines on plots $(a)$ and $(b)$ show plane intersects. Grey shading shows negative $u$ fluctuations (see key).

fluctuations (positive fluctuations are set to white). Together these plots illustrate some of the recognized emergent features in the log region. The horizontal plane of figure 1(a) clearly exhibits the spanwise stripiness noted by Ganapathisubramani et al. (2003) and Tomkins $\&$ Adrian (2003). For this $\log$ region view $(z / \delta=0.087)$, elongated low-momentum regions persist for the entire streamwise length of the viewing window, with signs of spanwise repetition (Hutchins, Ganapathisubramani \& Marusic 2004, $2005 a$ analysed spanwise repetition of these large-scale features by notch-filtering the PIV data). The simultaneous side view of plot (b) demonstrates that these features have a wider three-dimensional structure, extending some considerable distance in the wallnormal direction. The inclined plane of figure $1(d)$ is not simultaneously acquired, but shows a cross-stream slice through similar features. In this view the previously noted stripiness appears as tall low-momentum eruptions. Vortical structures accompany these low-momentum zones in arrangements that are, in some sense, consistent with the hairpin packet model (noted in various planes by Adrian et al. 2000; Tomkins \& Adrian 2003; Ganapathisubramani et al. 2003; Hutchins et al. 2005b; Hambleton et al. 2006; Marusic \& Hutchins 2006). Instantaneous realizations from DNS indicate rather more complex and tangled arrays of vortex cores, yet still clustered in or about these low-speed regions (Tanahashi et al. 2004; Jiménez \& del Álamo 2004). In all cited cases high momentum fluid has been noted to fill the regions between the low momentum stripes. Together these features dominate the two-point correlations of 

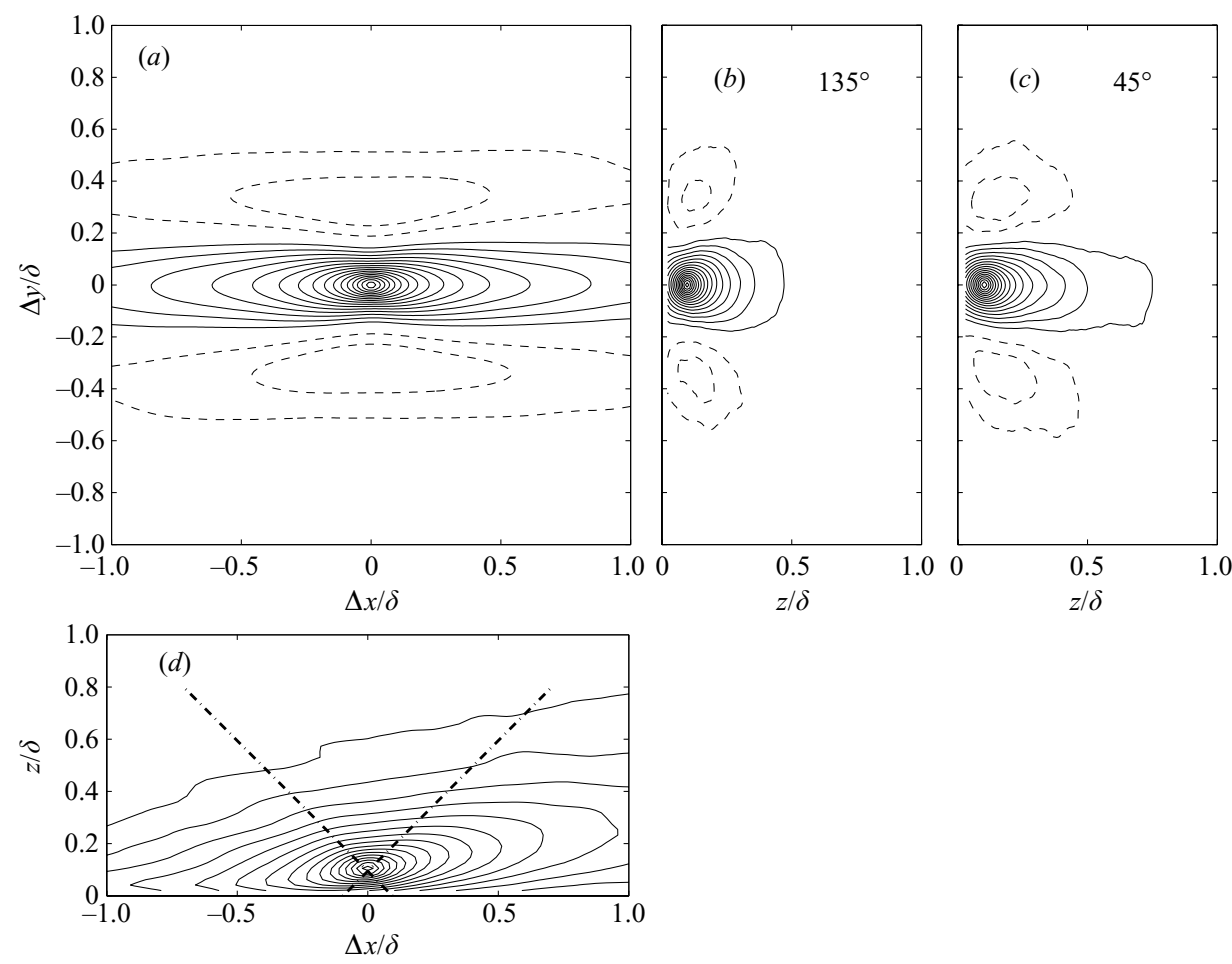

FIGURE 2. Two-point correlations of the streamwise velocity fluctuation $R_{u и}$ calculated at $z_{\text {ref }} / \delta=0.087$ for $(a)$ horizontal and $(d)$ vertical planes from combined plane experiments (b) $135^{\circ}$ and (c) $45^{\circ}$ inclined plane PIV. Contour levels are from $R_{u u}=-0.12$ to 0.96 in increments of 0.06 . Solid lines show positive contours and dashed lines show negative contours.

the streamwise velocity fluctuations $\left(R_{u u}\right)$ throughout the log region. Figure 2 shows iso-contours of $R_{\text {ии }}$ at $z_{\text {ref }} / \delta=0.087$ (for the same PIV data). Elongated positive correlation regions are flanked in the spanwise direction by anti-correlated behaviour (dashed contours), reflecting the spanwise stripiness of high- and low-momentum regions previously noted in figure 1 . It is clear from figure $2(d)$ and from the $45^{\circ}$ and $135^{\circ}$ comparison of plots $(b)$ and $(c)$ that the correlated regions are inclined in the downstream direction.

\section{Hot-wire rake results}

For the instantaneous flow fields and the correlation maps shown above, the length of the log-region structures exceed the PIV field-of-view. The rake measurements were undertaken in an attempt to redress this shortcoming and ascertain the true streamwise length of these features.

\subsection{Two-point correlations}

Figure 3 shows the outer-scaled two-point correlation of streamwise velocity fluctuation, $R_{u u}\left(\Delta x, \Delta y, z_{r e f}\right)$, for the rake experiments. The left-hand side shows the correlations in the streamwise direction at $\Delta y=0$. This is the autocorrelation, or the streamwise slice at $\Delta y=0$ through a correlation map of the type shown in figure 2(a). The right-hand side shows the spanwise variation of $R_{u u}$. For all available Reynolds numbers, spanning well over a decade in $R e_{\tau}$, there is a good collapse in the outerscaled $R_{u u}$. This is all the more striking given the large variation in $\delta$ across the 

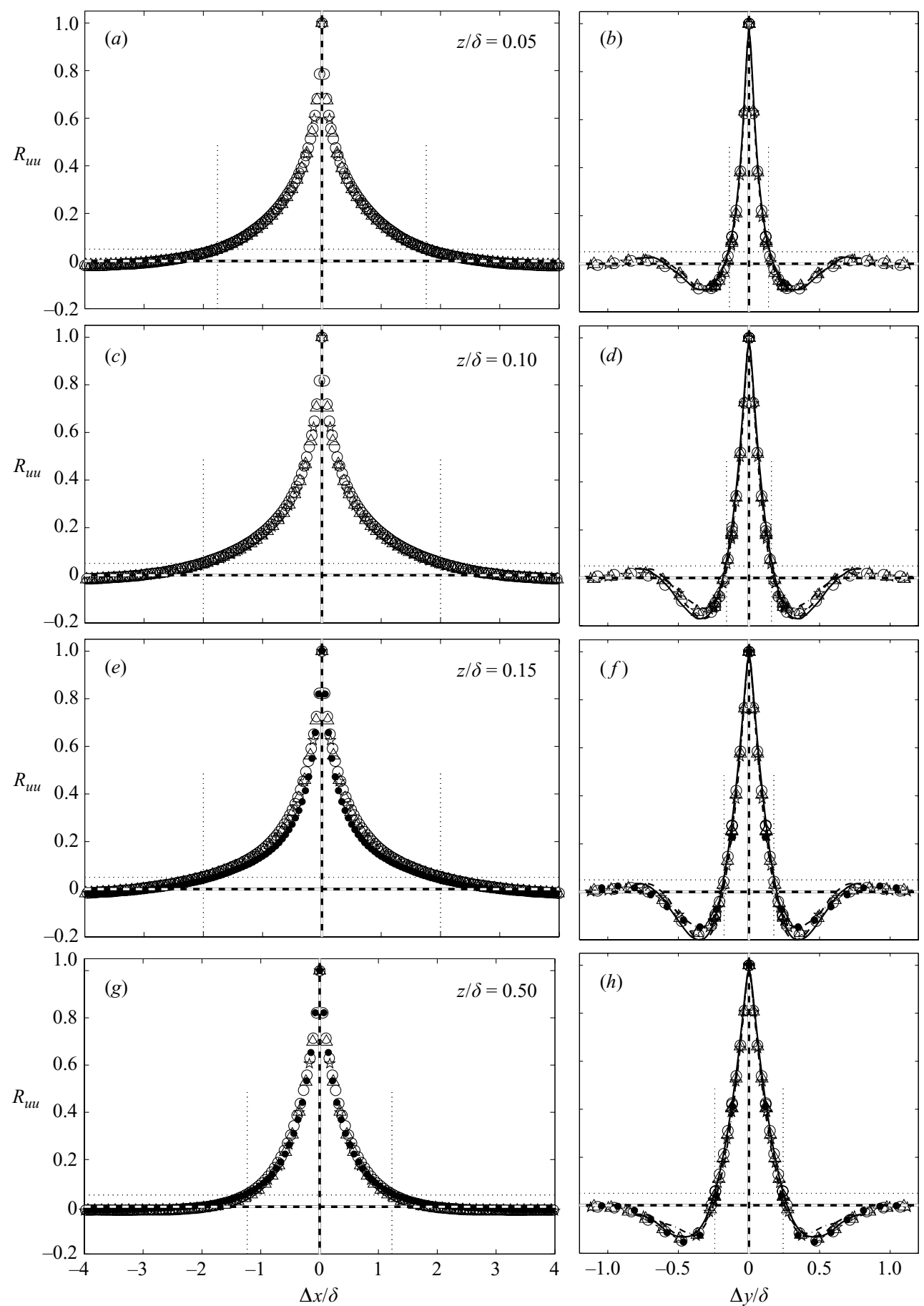

FIGURE 3. Streamwise (left) and spanwise (right) two-point correlations of the streamwise velocity fluctuation $R_{u u}$ calculated at $(a, b) z_{r e f} / \delta=0.05 ;(c, d) z_{r e f} / \delta=0.10 ;(e, f) z_{r e f} / \delta=0.15$; $(g, h) z_{\text {ref }} / \delta=0.50$. Symbols show rake data: $(\bullet) R e_{\tau}=1120 ;(\bigcirc) R e_{\tau}=7610 ;(\triangle) R e_{\tau}=14380$; (仿) $R e_{\tau}=19960$. Lines show $45^{\circ}$ inclined plane PIV results (dash-dotted) $R e_{\tau}=1010$; (dashed) $R e_{\tau}=1840$; (solid) $R e_{\tau}=2800$. 


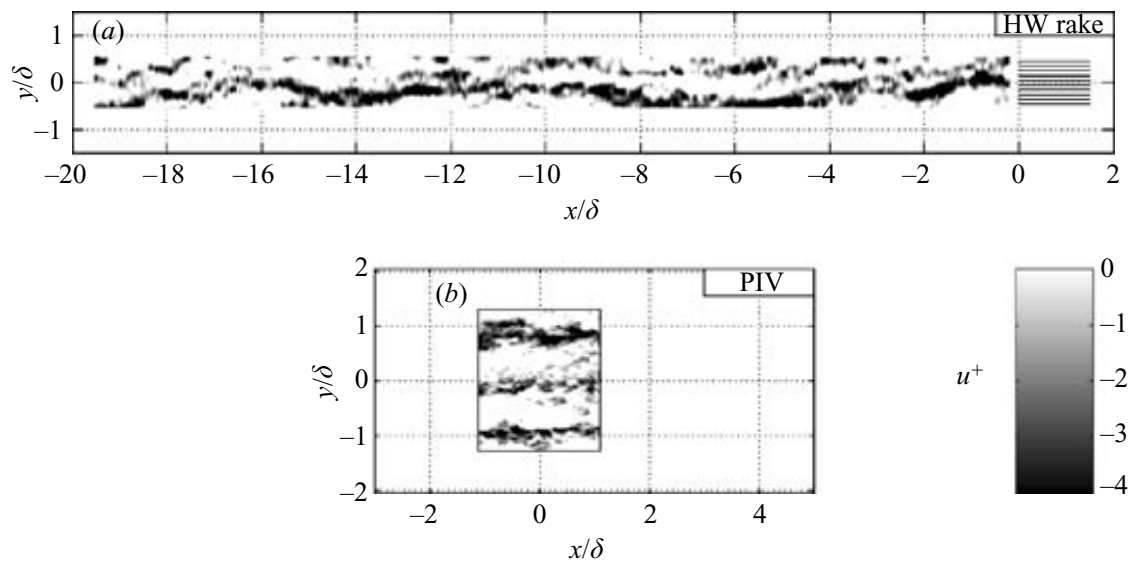

Figure 4. (a) Example of rake signal at $z / \delta=0.15$ for $R e_{\tau}=14380$. The $x$-axis is reconstructed using Taylor's hypothesis and a convection velocity based on the local mean, $\bar{U}=15.9 \mathrm{~m} \mathrm{~s}^{-1}$. (b) Typical PIV frame for comparison at $R e_{\tau}=1100, z / \delta=0.087$. Shading shows only negative $u$ fluctuations (see grey-scale).

experiments (from 0.069 to $0.336 \mathrm{~m}$ ). Outer scaling of correlation contours in the log and wake regions has also been observed by McLean (1990), Wark et al. (1991) and Hutchins et al. (2005b). The correlation profiles from the inclined plane PIV of Hutchins et al. (2005b) are included for comparison on the right-hand plots of figure 3. There is some indication of a low Reynolds number effect for the rake and PIV data at Reynolds numbers close to $R e_{\tau} \approx 1000$. At these Reynolds numbers the streamwise length of the correlations seems to be slightly shorter (solid symbols on figure $3 e$ ) and the anti-correlated regions seem less pronounced (dot-dashed lines on plots $b, d, f$ and $h$ ). Certainly at $R e_{\tau} \sim 1000$ the $\log$ region could still be considered as 'developing', with very little scale separation between the near-wall structure and the $\log$ region scales. $\dagger$ Regardless, the correlations scale well on boundary layer thickness as we move to higher $R e_{\tau}$. Comparing the plots on the left-hand side of figure 3 from top to bottom reveals that the streamwise length of the correlations increases from the wall up to the approximate centre of the log region (this length is later shown in $\S 6$ to peak at $z / \delta \approx 0.06$ ), with a pronounced shortening occurring in the outer wake region $(z / \delta=0.50)$. To highlight this effect the dotted lines on figure 3 show the size of the positive correlated regions at $R_{u u}=0.05$. Considering the right-hand side of figure 3 , we note that the spanwise width of the correlation region increases approximately linearly with $z / \delta$ for all heights investigated, as was noted for the inclined-plane PIV by Hutchins et al. (2005b).

\subsection{Instantaneous data}

The streamwise extent of the log region structures has been previously inferred from single-point statistics such as those shown on the left-hand side of figure 3 . The long tails in the autocorrelation curves and the peak in the premultiplied spectra $k_{x} \Phi_{u u}$ have consistently indicated length scales $O(6 \delta)$ for turbulent boundary layers. In reality, when raw fluctuating velocity signals from the rake are viewed, we commonly see features that are far in excess of these length scales. Figure 4(a) shows a sample

$\dagger$ If we take the start of the $\log$ region to be $z^{+}=100$ and the upper limit to be $z / \delta=0.15$, the extent of the $\log$ region at $R e_{\tau}=1120$ is minimal (spanning only $0.06 \delta$ ). 
segment from the rake at $z / \delta=0.15$. A very long meandering feature appears to snake through the measurement domain for over $20 \delta$. For the Reynolds number shown $\left(R e_{\tau}=14380\right)$ this amounts to a physical length of over $6.5 \mathrm{~m}$. This implies that the log region stripiness previously observed from PIV data is actually a snapshot of a far larger structure (see the PIV comparison in figure 4b). Furthermore, although the low-speed region in figure $4(a)$ is clearly very long, the meandering tendency will mask its true length from single-point statistics. A synthetic model flow is used in $\S 4.3$ to demonstrate this. It should be clarified that the feature shown in figure 4 is by no means unusual and is typical of much of the rake data we have obtained. At the present time it is impossible for us to compile accurate length scale and frequency statistics since, for the most part, these features are rarely fully contained within our measurement domain, and instead tend to wander into and out of our limited spanwise domain due to meandering. All we can really state at this stage is that such events are prevalent features of the log region at all Reynolds numbers we have tested (this is reinforced by the DNS and ASL measurements of $\S \S 5$ and 7).

\subsection{Synthetic flow fields}

The log region is modelled with a simple large-scale streak-type structure, consisting of elongated low-speed regions flanked on either side by high-speed regions of similar length and width. The spanwise width of the low- and high-speed regions $\left(L_{y}\right)$ is set to $0.4 \delta$, chosen to reflect the scales observed from instantaneous rake and PIV data (and also the approximate width of the correlation regions in figures 2 and 3). Here we refer to case I as that where the elongated features are perfectly aligned with the mean flow direction, as shown in figure 5(a). Case II corresponds to the same but with an imposed low-frequency spanwise sinusoidal meander, with peak-to-peak amplitude $A=0.4 \delta$ and period $\omega=12 \delta$ (a detailed description of these synthetic flows is given in Appendix A). The entire signal is modulated in the streamwise direction by a Hanning window of length $L_{x}$, forcing the fluctuations at either end to zero. Figures $5(a)$ and $5(b)$ show the final functions for cases I and II respectively. To create the full synthetic flow fields, the aligned or meandering features are randomly arranged with a normal distribution of length scales, as described in Appendix A.

Figure 5(c) shows the resulting auto-correlation curves for the two cases. Although identical length scales are modelled in each case, the meandering tendency of case II has substantially shortened the length of the positive correlation region. The zero-crossings of the flow-aligned case (solid) approximately reflect the maximum streamwise length of the distributed features. For the meandering case (dashed), the shortened correlation becomes negative at approximately $\Delta x \approx \pm 3.5 \delta$, considerably shorter than its true length. Figure $5(d)$ shows the one-dimensional pre-multiplied streamwise energy spectra in the $x$ direction $\left(k_{x} \Phi_{u u}\right)$ for both synthetic flow fields. Once again, it is very clear that the spanwise meandering can lead to a misinterpretation of the true length scale when considering only single-point statistics such as energy spectra.

Additional comments on the potential pitfalls of using energy spectra to infer structural characteristics are further discussed in Appendix B. In the meantime, since we have observed meandering of large-scale features in the instantaneous rake data (figure 4), a more detailed comparison is made with the statistics generated by the meandering synthetic flow field. The anti-correlated regions that occur beyond $\Delta x \approx \pm 3.5 \delta$ for the meandering case (II) in figure 5(c) have also been observed in the experimental rake data. Zero-crossings are noted in figure $3(a, c, e)$, although the resulting regions of anticorrelation are somewhat weaker. The meandering fake 


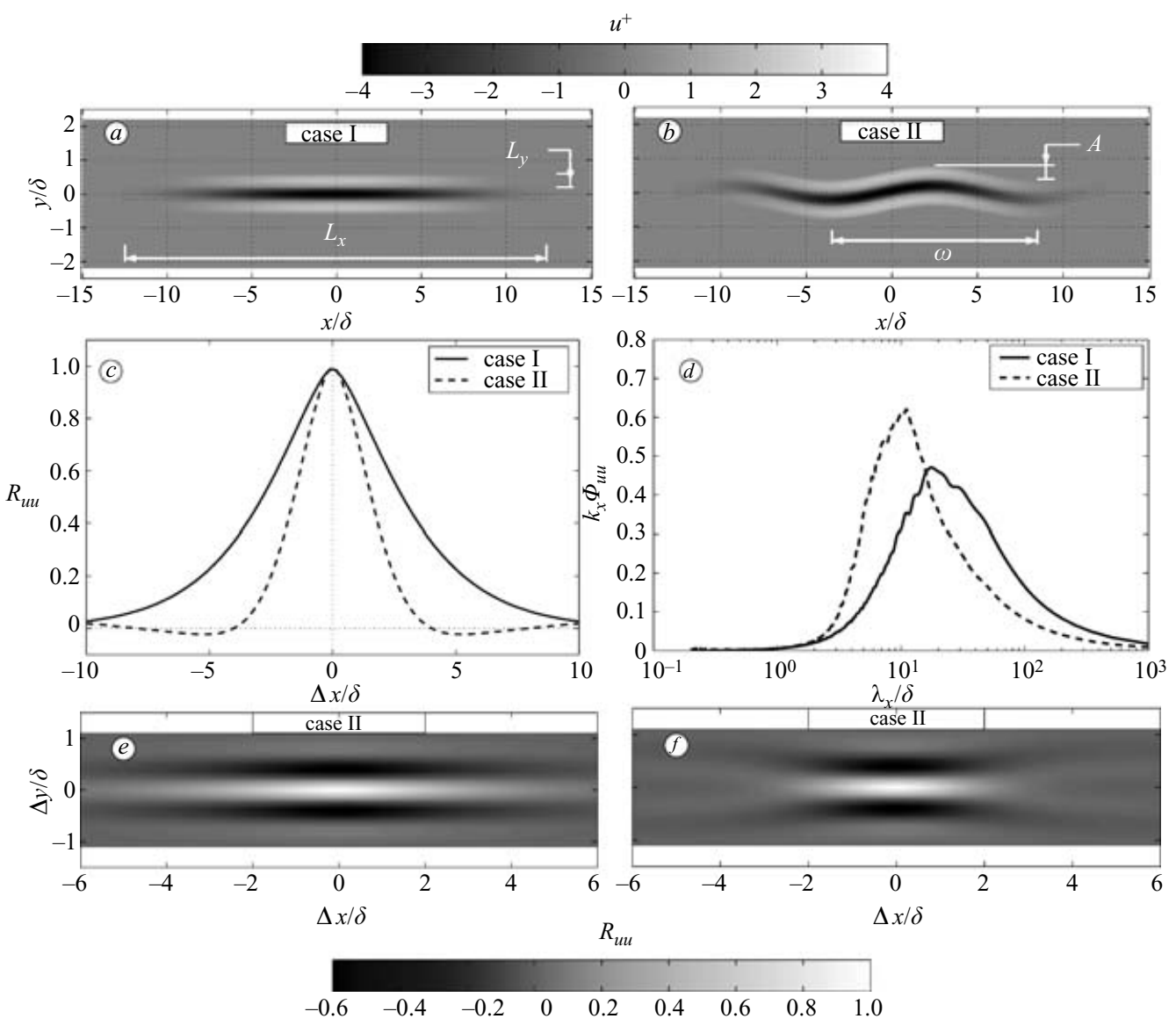

FIGURE 5. The log region modelled as elongated large-scale stripy structures. (a) Case I, streamwise-aligned. (b) Case II, meandering with amplitude and period of 0.6 and $12 \delta$ respectively. Grey scale shows velocity fluctuation (see key). (c) Auto-correlations for a random array of each modelled flow. $(d)$ Corresponding premultiplied energy spectra (solid) case I; (dashed) case II. The streamwise/spanwise two-point correlation maps of $R_{u и}$ are given for (e) case I, $(f)$ case II. Grey scale shows $R_{\text {ии }}$ level (see key).

features lead to an interesting streamwise/spanwise correlation map beyond the zero-crossing points. These $R_{u u}$ maps are shown for cases I and II in figures 5(e) and $5(f)$. As well as being substantially shorter in the streamwise direction, the meandering case (plot $f$ ) yields a distinctive ' $\mathrm{X}$ '-shaped pattern of positive correlation, with anticorrelated regions arranged up- and downstream of the reference point at $\Delta x \gtrsim \pm 3.5 \delta$. Interestingly, we see signs of such a pattern when we construct a similar $R_{u u}$ map from the experimental data, shown in figure 6. Though the effect is weaker (the greyscale increment of figure 6 is altered to highlight this), we note the same distinctive pattern occurring beyond the zero-crossings. The case shown is for the $R e_{\tau}=14380$ experiment at $z / \delta=0.05$, but these features are evident for all Reynolds numbers and at all measurement heights within the log region. The qualitative similarity between figure 5(e) and figure 6 would seem to reinforce our initial observations that the very long features in the log region meander appreciably, and that this hides their true length from single-point statistics. 


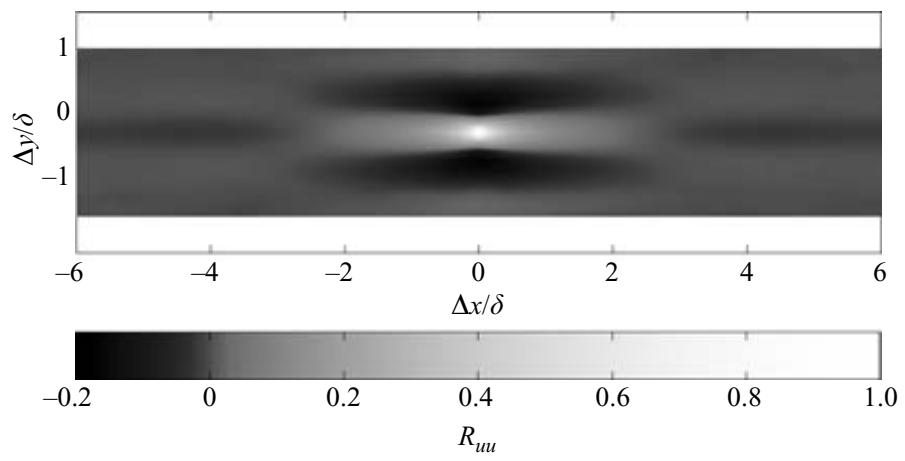

FIGURE 6. Streamwise/spanwise two-point correlation map of $R_{u u}$ at $z / \delta=0.05$ for $R e_{\tau}=14380$. Grey-shading shows $R_{u и}$ level (note nonlinear increments: see key).

\section{Evidence from direct numerical simulations}

Large numerical domain DNS has also uncovered evidence of very large-scale features inhabiting the log region of wall-bounded shear layers. Figure 7 shows streamwise velocity fluctuations from recent channel flow simulations of del Álamo et al. (2004) at $R e_{\tau}=934$. Plot $(a)$ shows the $u$ fluctuations in the log region at $z^{+}=150(z / h=0.164$, where $h$ is the channel half-height $)$. Clearly visible in this plot are several occurrences of very long regions of negative $u$ fluctuation, visible in the plot as elongated dark regions. (In separate DNS studies Tanahashi et al. 2004 show complex clusters of fine-scale vortices existing within these elongated lowspeed features.) These features are $>10 \mathrm{~h}$ in length and have characteristic length scales that scale on outer variables (as shown on the earlier correlation plots). For boundary layers, the streamwise and spanwise length scales inferred from the peak in $k_{x} \Phi_{u u}$ and $k_{y} \Phi_{u u}$ are of the order $\lambda_{x} \approx 6 \delta$ and $\lambda_{y} \approx 0.7 \delta$ respectively. Different length scales have been reported for channel and pipe flows (Jiménez 1998; Kim \& Adrian 1999). Figure 7(b) shows the corresponding streamwise velocity fluctuations at $z^{+}=15(z / h=0.016)$. In this near-wall region, inner-scaled streaks dominate with commonly reported characteristic length scales $\lambda_{x}^{+} \approx 1000$ and $\lambda_{y}^{+} \approx 100$ (e.g. Jiménez $\&$ del Álamo 2004). As well as illustrating these two scales, figure 7 seems to imply some kind of interaction. If we peer through the small inner-scaled structures of plot $(b)$, we can see a faint superimposed footprint of the larger-scale features. This characteristic is highlighted by applying a filter of size $(h / 2 \times h / 2)$ to both planes of data. Here, the aim of the filter is to average away the small-scale features, and for this purpose a simple Gaussian is adequate. Figure $7(c)$ and $(d)$ shows just the negative streamwise velocity fluctuations as grey-scale contours for the filtered fields, and it is noted that remarkable similarities now appear between the remaining large-scale events for both wall-normal positions. One such feature is highlighted by the dot-dashed contour on figure $7(c)$, which is drawn at a small negative value $\left(u^{+}=-0.2\right)$. This feature exceeds the length of the numerical domain $(>25 h)$. When the same contour is plotted on figure $7(d)$, with an appropriate streamwise shift, we note that it encloses almost exactly the same large-scale feature. If we refer back to the original velocity fields of plots $(a)$ and $(b)$ it is possible to discern this large-scale feature even through the unfiltered data. In separate DNS studies, Abe, Kawamura $\&$ Choi (2004) have also noted a footprint from the outer-layer structure onto the near-wall region, observing that these large-scale structures contribute to the shearstress fluctuations. Tsubokura (2005) concluded a similar result from LES studies of 

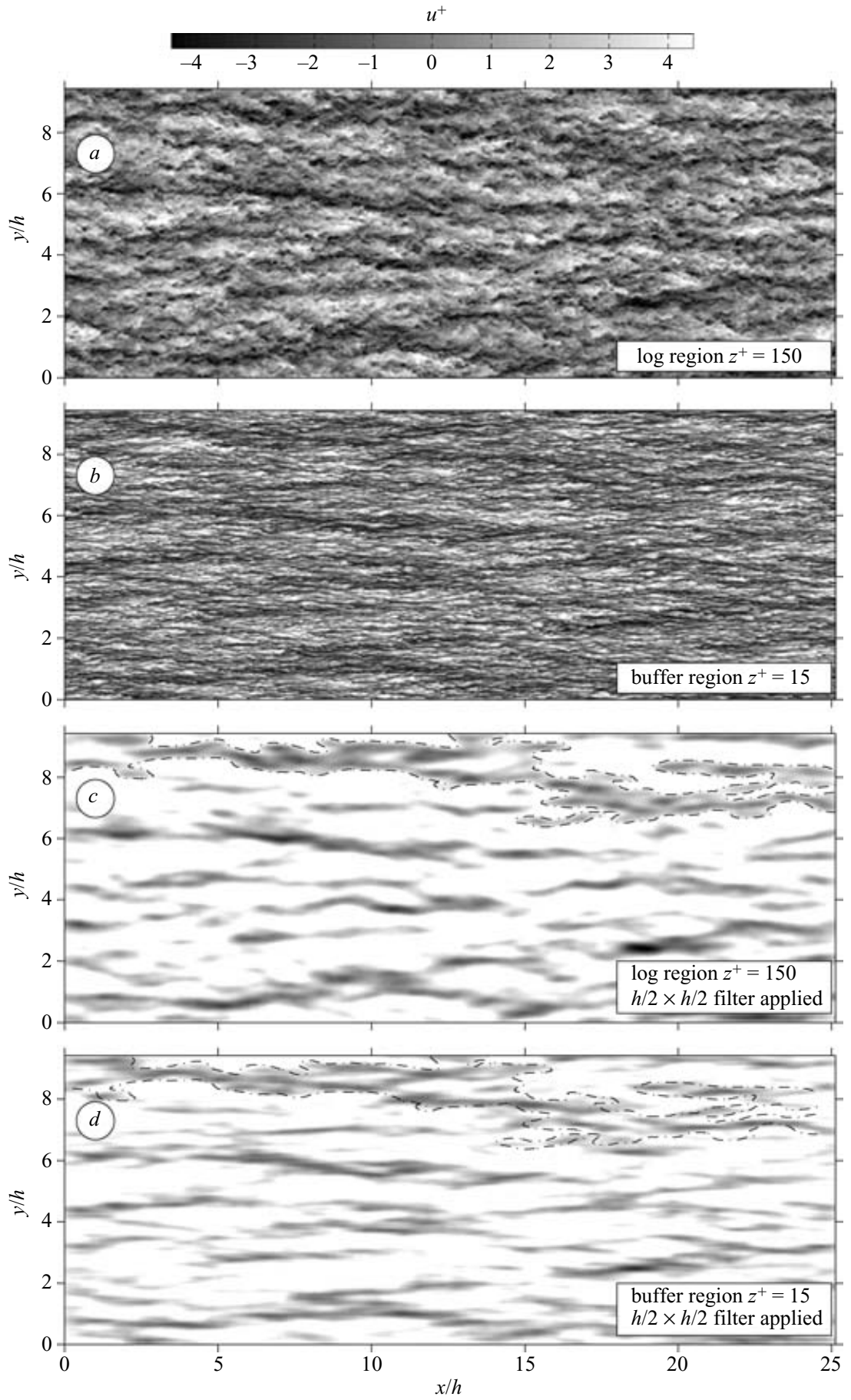

FigURE 7. Instantaneous flow fields from $R e_{\tau}=934$ channel simulations (del Álamo et al. 2004). Shading shows $u$ fluctuations $(a)$ at $z^{+}=150 ;(b)$ at $z^{+}=15$ (see upper grey-scale). Plots $(c)$ and $(d)$ show the filtered data at the same respective heights (shading shows negative $u$ fluctuations, grey-scale as figure 4). 
pipe and channel flows. Hutchins et al. (2005b) have shown previously that the largescale $\log$ and wake region events retain a measurable correlation with the near-wall buffer region ('attached' and 'detached' correlation regimes), and by such mechanisms very low wavenumber energy leaches into the near-wall region. This conclusion is well supported by the result in figure 7, and is consistent with the finding that the streamwise turbulence intensity in the viscous sublayer and buffer region will have a $\delta$-scale influence (see for example DeGraaff \& Eaton 2000; Metzger et al. 2001; Metzger \& Klewicki 2001; Marusic \& Kunkel 2003; Jiménez, del Alamo \& Flores 2004). Further implications to Reynolds number scaling will be considered in $\S 6$.

The mechanisms that give rise to the large-scale features in the log region are somewhat unclear at this stage. Figure 7(b) seems to show that the small inner-scaled nearwall features are arranged into larger outer-scaled agglomerations, possibly by the footprint of the log region structures. We should not, however, exclude the possibility that there is a feedback mechanism by which the modulated near-wall events influence the large-scale structures (although such scenarios become increasingly difficult to countenance at high Reynolds number, where the separation in scale between these inner- and outer-scaled events becomes very large see $\S 7$ ). The autonomous channel simulations of Jiménez et al. (2004) would seem to imply that the near-wall structures can self-organize into very long features even in the absence of the outer scales, and Jiménez \& del Álamo (2004) attribute the very long $u$ fluctuations in the log region to 'passive wakes' formed downstream of smaller attached clusters of vortices originating from the buffer region. They suggest that these 'wakes' spread under the effect of background turbulence. However, it is worth noting that a similar-looking 'spreading wake' can be obtained by conditionally averaging on meandering features such as those shown in figure 5. Furthermore, in an instantaneous sense, neither the PIV nor the DNS data exhibit any signs of a widening of these large-scale features in the streamwise direction (see figures $1 b, 4 a$ and $7 a \dagger$ ). In further DNS studies, Toh \& Itano (2005) also note near-wall scales existing under large-scale structures and suggest that these two disparate scales interact in a co-supporting cycle. Certainly, figure 7 implies a connection between the two scales. As a final important point, the authors have recently noted that the very long log region structures exist even over fully roughened walls, which would imply that the near-wall cycle is not responsible for their formation.

\section{Reynolds number effects}

It is now well known that the inner-scaled peak in the streamwise turbulence intensity rises with Reynolds number. One of the first clear examples of this is given by DeGraaff \& Eaton (2000) and Metzger \& Klewicki (2001), who collated streamwise turbulence intensity results from hot-wire measurements where the nondimensional wire length was less than 10 wall units (to avoid any possibility of spatial resolution issues). The collated results convincingly demonstrate that the peak rises with Reynolds number. Since that time, similar results have been presented by Marusic \& Kunkel (2003) and Jiménez \& del Álamo (2004). Figure 8 replots the data from Metzger \& Klewicki (2001), with the addition of numerical results and the similarity approximations of Marusic \& Kunkel (2003). In general, figure 8

$\dagger$ If there were substantive widening of flow features in the streamwise direction, it would be simple to deduce flow direction without reference to the axis system in the streamwise/spanwise planes of figures $1(b), 4(a)$ and $7(a)$. This is not the case (flow is from left to right). 


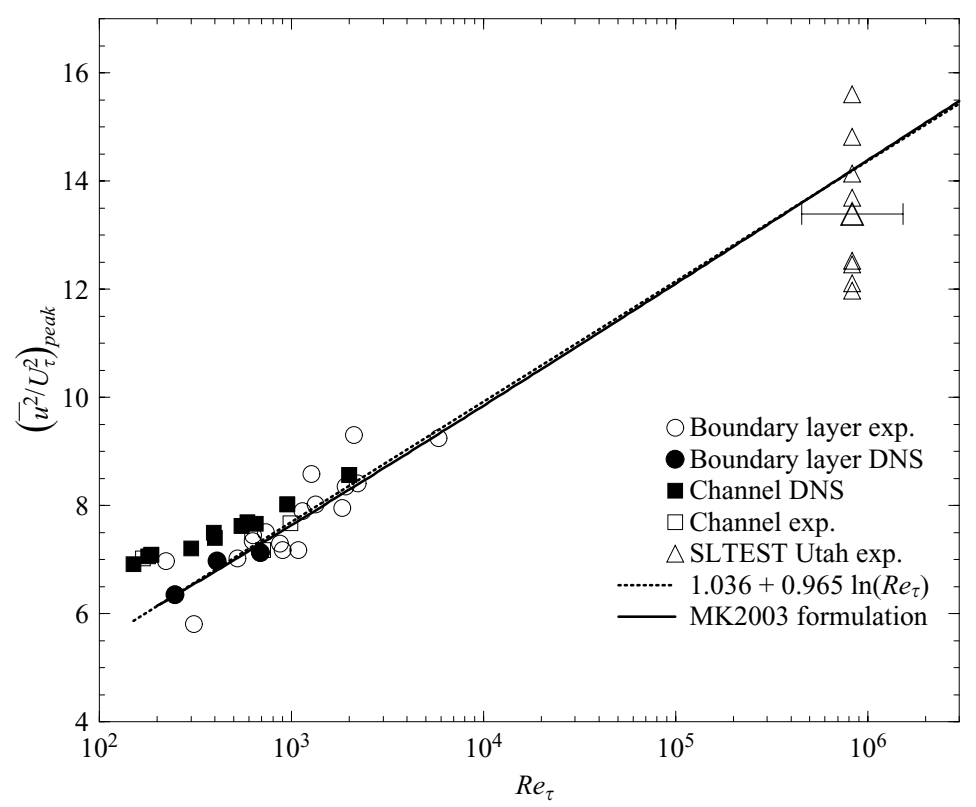

\begin{tabular}{|c|c|c|c|}
\hline Authors & $R e_{\tau}$ & Flow & Technique \\
\hline DeGraaff \& Eaton (2000) & $734,1335,2217,5813$ & BL & $\exp$ \\
\hline Ueda \& Hinze (1975) & 633,1918 & BL & $\exp$ \\
\hline Ligrani \& Bradshaw (1987) & 1140 & BL & $\exp$ \\
\hline Balint et al. (1991) & 1088 & BL & $\exp$ \\
\hline Johansson et al. (1987) & 1845 & BL & $\exp$ \\
\hline Johansson \& Karlsson (1989) & 900 & BL & $\exp$ \\
\hline Ching et al. (1995) & 222,627 & BL & $\exp$ \\
\hline Purtell et al. (1981) & 311,870 & BL & $\exp$ \\
\hline$?$ & $522,1269,2109$ & BL & $\exp$ \\
\hline Spalart (1988) & $248,410,690$ & BL & DNS \\
\hline Wei \& Willmarth (1989) & $169,714,989$ & $\mathrm{CH}$ & $\exp$ \\
\hline Jiménez and coworkers & $186,547,934,2003$ & $\mathrm{CH}$ & DNS \\
\hline Moser et al. (1999) & $180,395,590$ & $\mathrm{CH}$ & DNS \\
\hline Iwamoto et al. (2002) & $110,150,300,400,650$ & $\mathrm{CH}$ & DNS \\
\hline Metzger \& Klewicki (2001) & $8 \times 10^{5}$ & ASL & $\exp ($ SLTEST) \\
\hline
\end{tabular}

FIGURE 8. Variation of the peak value of the inner-scaled streamwise turbulence intensity with Reynolds number (around $z^{+} \approx 15$ ). $\square$, channel flow; $\bigcirc$, boundary layer; $\triangle$, atmospheric surface layer. Solid symbols are DNS results, open are experimental. MK2003 refers to Marusic \& Kunkel (2003).

reinforces the same trend with Reynolds number, although there is some suggestion that channel flows (square symbols) may behave differently to boundary layers at the low Reynolds number end. The dashed line shows the similarity approximation of Marusic \& Kunkel (2003), which describes well the available boundary layer data. A simple curve fit to their result is given by

$$
\left(\frac{\overline{u^{2}}}{U_{\tau}^{2}}\right)_{\text {peak }}=1.036+0.965 \ln \left(R e_{\tau}\right) .
$$




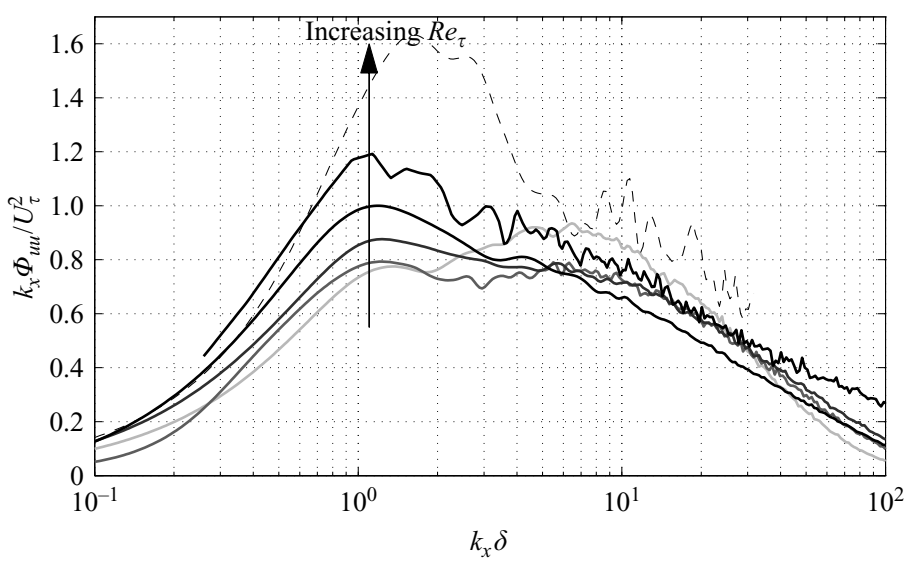

FIGURE 9. Pre-multiplied energy spectra $k_{x} \Phi_{u u} / U_{\tau}^{2}$ at $z / \delta=0.06$ for laboratory hot-wire measurements. Line shading indicates Reynolds number. From lightest to darkest $R e_{\tau}=1010$, 1910, 2630, 4110 and 7300. Dashed line shows sonic anemometer data from SLTEST (Utah 2005) at $\operatorname{Re}_{\tau} \approx 6.6 \times 10^{5}, z / \delta=0.06$.

By low-pass-filtering the high Reynolds number data, Metzger \& Klewicki (2001) show that the additional energy in the near-wall streamwise velocity fluctuations comes from low-wavenumber events. Figure 7 clearly shows the origins of the lowwavenumber energy, which is present in the near-wall region in the form of a footprint from the very large outer-scaled log-region structures. In order to consider the scaling of the low-wavenumber contributions, figure 9 shows pre-multiplied spectra scaled with $U_{\tau}^{2}\left(k_{x} \Phi_{u u} / U_{\tau}^{2}\right)$ for various laboratory hot-wire measurements at $z / \delta=0.06$, made in the range $1010 \leqslant R e_{\tau} \leqslant 7300$ (experiments are described in $\S 2$ and table 2 ). For these data it is noted that a strengthening peak emerges in the pre-multiplied spectra with Reynolds number, centred around $k_{x} \delta \approx 1$, and increasing in magnitude with $R e_{\tau}$. Clearly a Reynolds number dependence is evident, indicating that the large-scale structures in the log region may not scale exclusively on $U_{\tau}$. If it can be established that the large-scale structures are caused by the local shear in the log region then the velocity difference across this region might be a suitable candidate. However, this remains unclear and further study is required to resolve the velocity scaling issue for the largest structures. In particular, high Reynolds number data are needed to confirm the trend with increasing $R e_{\tau}$. As an example, a preliminary result from the atmospheric surface layer is included in figure 9 as a dashed line. Though this indicates a continuing $R e_{\tau}$ dependency, some caution is required owing to uncertainty over convergence. This measurement was obtained from 1 hour of sonic anemometer data during periods of steady wind and neutral buoyancy on 2 June 2005 (see $\S 7$ ).

To gain a better insight into the contributions to the $u$ spectra across the boundary layer, figure 10 shows colour contours of $k_{x} \Phi_{u u} / U_{\tau}^{2}$ versus $\log \left(\lambda_{x} / \delta\right)$ across all measured wall-normal positions, together with the corresponding mean velocity and turbulence intensity profiles. Plots $(a)$ to $(d)$ represent increasing Reynolds number, with $R e_{\tau}=1010,1910,2630$ and 7300 respectively. The figure shows two distinct energy peaks emerging with increasing Reynolds number. For convenience we will refer to these as the 'inner' and 'outer' sites. The inner site is fixed in viscous coordinates, occurring at $z^{+} \approx 15$ and $\lambda_{x}^{+} \approx 1000$ (shown by the white + symbol). For the Reynolds numbers shown here this is the strongest peak, and occurs at the same wall-normal location as the peak in the broadband turbulence intensity (shown by the filled circles). 


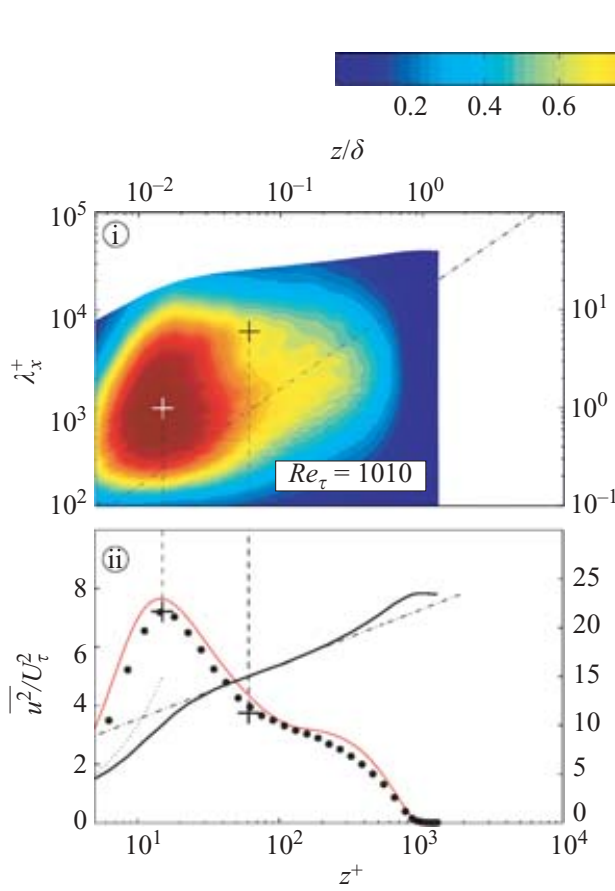

(a)
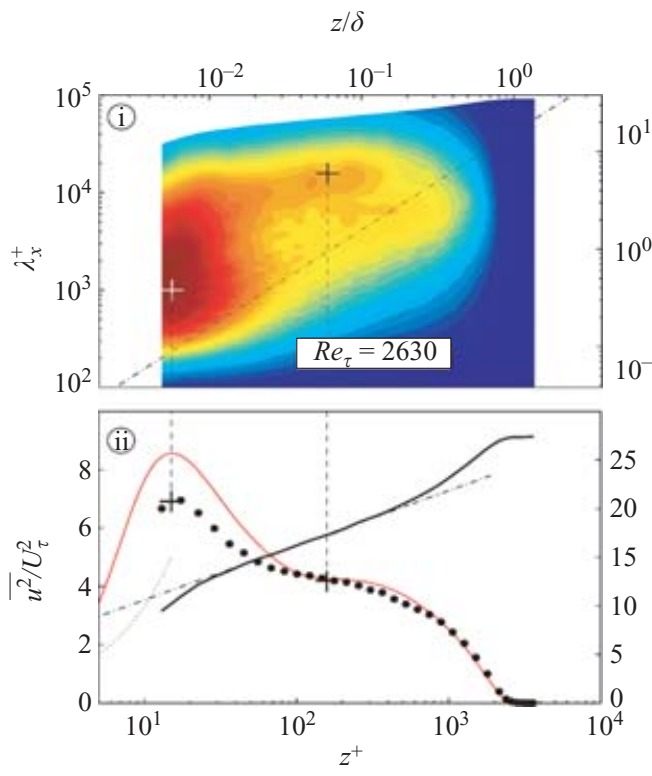

(c)
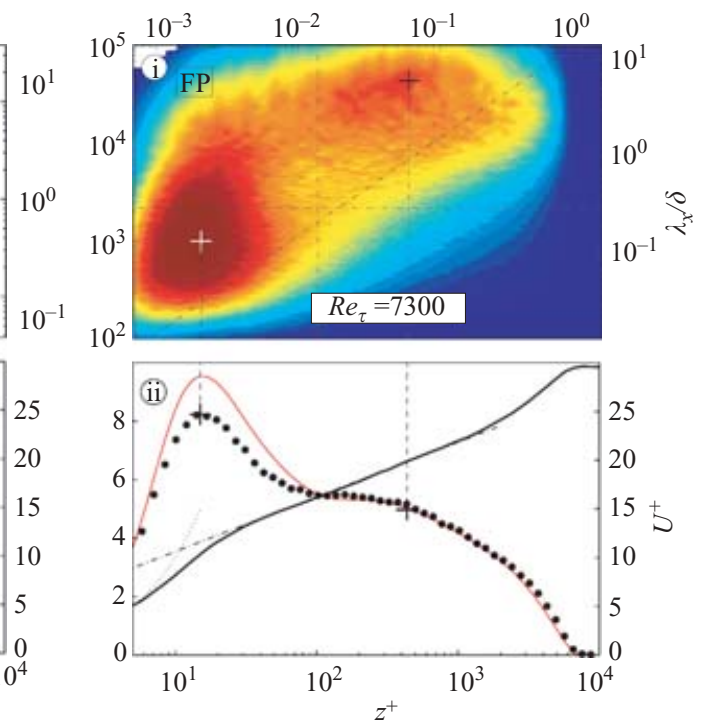

(d)

FIGURE 10. (i) Contour maps showing variation of one-dimensional pre-multiplied spectra with wall-normal position for (a) $R e_{\tau}=1010$; (b) $R e_{\tau}=1910$; (c) $R e_{\tau}=2630$; (d) $R e_{\tau}=7300$. The $y$-axis shows length scale in both inner (left) and outer (right) scaling. The $x$-axis shows wall-normal position for both inner (lower) and outer scaling (upper). The colour scale shows magnitude of $k_{x} \Phi_{u u} / U_{\tau}^{2}$. (+) denote 'inner' and 'outer' energy sites (white) $\lambda_{x}^{+}=1000, z^{+}=15$ and (black) $\lambda_{x}=6 \delta, z=0.06 \delta$. Dot-dashed lines show $k_{x}^{-1}$ limits $\lambda_{x}=15.7 z$ (and $z^{+}=100$ and $\lambda_{x}=0.3 \delta$ for plot $d$ ). (ii) Corresponding mean profiles of $(\bullet)$ turbulence intensities and $(-)$ velocity. Dotted line shows $u^{+}=z^{+}$. Dashed lines and $(+)$symbols denote $z^{+}=15$ and $z=0.06 \delta$. Dot-dashed line shows $U^{+}=(1 / \kappa) \ln \left(z^{+}\right)+A$ (where $\kappa=0.41$ and $\left.A=5.0\right)$. Red line shows MK2003 formulation. 
This inner site is due to the near-wall cycle of streaks and quasi-streamwise vortices (Kline et al. 1967; Jiménez \& Pinelli 1999; Schoppa \& Hussain 2002). As Reynolds number increases, the second (outer) energy site emerges (shown by the black + in figure 10). This occurs within the logarithmic region of the mean velocity profile and is approximately fixed in outer coordinates at $z / \delta \approx 0.06$ and $\lambda_{x} / \delta \approx 6$ (although there is some indication that the precise location of the peak wanders gradually with Reynolds number). The energy in this outer site comes from the largest modes in the turbulent boundary layer that we have here termed 'superstructures'. Namely, the very long regions of meandering positive and negative $u$ fluctuation inhabiting the logarithmic region, as evidenced by the PIV, hot-wire rake and DNS data of figures 1, 4 and 7 respectively. The size of these events scales on $\delta$, and their magnitude primarily on $U_{\tau}^{2}$ but with a clear $R e_{\tau}$ dependence. A cursory glance at the increasing Reynolds number cases of plots $(a)$ to $(d)$ gives a very vivid impression of the emergence of the outer energy site with $R e_{\tau}$. At the lowest Reynolds number, there is insufficient separation of scales to observe the outer peak and the energy from the inner-scaled events swamps the weaker log region structure. The plots indicate that Reynolds numbers of $R e_{\tau} \gtrsim 2000$ are required before we will clearly see an emergence of the outer site. It is also important to note from the higher Reynolds number cases of figure 10 that the emergent large-scale energy is felt all the way down to the wall (this region is labelled FP for footprint in figure 10d). This increasing presence of low-wavenumber energy in the near-wall region will likely contribute to the rising near-wall peak in the broadband turbulence intensity at $z^{+}=15$ (see figure 8 ). The low-wavenumber energy is essentially the footprint due to the log region structure imparting a large-scale organization or modulation on to the near-wall cycle (see figure 7). Del Alamo \& Jiménez (2003) decomposed $u$ fluctuations from $R e_{\tau}=550$ channel DNS into two plots of $k_{x} \Phi_{u u}$ where $\lambda_{y}>0.75 h$ and $\lambda_{y}<0.75 h$. They show similar trends of inner and outer energy sites to those shown in figure 10 (although to see this behaviour at low Reynolds numbers required segregation according to spanwise scale).

It is important to monitor the effect of limited spatial resolution for the spectra shown in figures 9 and 10. The same hot-wire probe was used for all Minnesota spectra and thus the non-dimensional wire length has varied from $l^{+}=14,30,40$ and 67 for $R e_{\tau}=1010,1910,2650$ and 4110 (see table 2). This can clearly be seen in the diminishing magnitude of the inner energy site in figure $10(a-c)$ at $z^{+} \approx 15, \lambda_{x}^{+} \approx 1000$. The $R e_{\tau}=7300$ measurements were made in a larger facility and are better resolved with $l^{+}=22$. For the results reported here, spatial resolution is not such an issue when considering the large-scale low-wavenumber phenomena in the logarithmic region of the boundary layer (figure 9). However, in the near-wall region, where the energycontributing motions are smaller, the limited resolution becomes a problem. The red lines on the profile plots of figure 10(ii) show the predicted turbulent intensities for each Reynolds number and thus highlight the discrepancies. These lines are from the similarity formulation of Marusic \& Kunkel (2003), which has been shown to compare well to other high spatial resolution data at these Reynolds numbers (such as those shown in figure 8).

From scaling arguments based on the attached eddy hypothesis (Townsend 1956; Perry, Henbest \& Chong 1986; Marusic \& Perry 1995) we would expect to see $k_{x}^{-1}$ behaviour spanning the region between the inner and outer energy sites for eddies that scale with their distance from the wall. A hairpin vortex packet scenario would be consistent with these attached eddies being mainly aligned and spatially arranged within the large superstructure (Marusic 2001), which is also consistent with VLSM of Kim \& Adrian (1999). Owing to the presence of the aforementioned inner and 
outer energy sites, significant regions of $k_{x}^{-1}$ behaviour are only possible for high Reynolds numbers very close to the wall. Nickels et al. (2005) estimated that a $k_{x}^{-1}$ region in the $u$-spectra will occur within the limits $k_{x} \delta>21 ; k_{x} z<0.4$ for $z^{+}>100$. Therefore a triangular $k_{x}^{-1}$ region would appear as a plateau on the premultiplied spectra plots in figure 10 bounded by

$$
z^{+}>100, \quad \lambda_{x} / z>15.7, \quad \text { and } \quad \lambda_{x} / \delta<0.3 .
$$

Based on the above limits the $k_{x}^{-1}$ region will not exist for $R e_{\tau}<5230$, and thus it is only on figure $10(d)$ at $R e_{\tau}=7300$ that a small $k_{x}^{-1}$ region appears. Even so, with increasing Reynolds number it is clear that the intermediate scales (between the inner and outer energy sites) in the log region do tend to follow the trend described by the $k_{x}^{-1}$ formulation (the line $\lambda_{x} / z=15.7$ is shown on each spectra plot in figure 10). Further high Reynolds number experimental data are needed to confirm this behaviour fully. Such experiments are also required to remove any uncertainty regarding the apparent strengthening of the 'superstructures' with increasing $R e_{\tau}$, as suggested by the data in figure 9. In this case the extent of a $k_{x}^{-1}$ region would be further reduced as the outer energy site encroaches on the limit $\lambda_{x} / \delta=0.3$.

Figure 11 is a sketch summarizing our interpretation of some salient features of the spectra shown in figure 10. In this simple model, the energy spectrum is composed of two main separate energy sites. Close to the wall, at the point of maximum turbulence production, the near-wall cycle causes an inner energy site with location and magnitude fixed in viscous units $\left(v\right.$ and $\left.U_{\tau}\right)$. In the logarithmic region the 'superstructures' lead to an outer energy site with location fixed on outer units $(\delta)$ and a magnitude that appears to rise with Reynolds number. As $R e_{\tau}$ increases, these two sites become increasingly separated. Co-existent in the logarithmic region is a region of $k_{x}^{-1}$ behaviour due to attached eddies that scale with distance from the wall $z$ and $U_{\tau}$. In addition, and not shown on figure 11, we would expect wake structure contributions beyond the edge of the logarithmic region due to eddies that scale with $\delta$ and $U_{\tau}$ (Perry \& Marusic 1995), although their contribution is relatively weak in zero pressure gradient flows.

Examples of the structure associated with inner and outer energy sites are included in figure 11 as grey-level contours of $u$ fluctuation. The data are from the channel flow DNS of del Álamo et al. (2004) and are included for illustrative purposes only. (The sketched spectrum corresponds to $R e_{\tau} \approx 4000$, while the DNS data are for $R e_{\tau}=934$.) These data highlight the inner/outer interaction, with energy from the outer site or 'superstructure' extending down to the near-wall region. This is evident in both the spectrum and the two $x-y$ planes of $u$ fluctuation, where the large-scale structure in the log region leaves a faint imprint on the near-wall region. The white contour highlights the same large-scale feature evident at both wall-normal locations (a large low-speed, darker-shaded region). The contribution of this footprint to the energy spectrum is shown by the arrows linking these two contours. As Reynolds number increases, the footprint in the near-wall region will become progressively more noticeable. This is not only due to the increasing scale separation between the inner and outer scales, but also because the magnitude of the 'superstructure' fluctuation becomes increasingly comparable to the (viscous-scaled) fluctuation due to the near-wall cycle.

As noted earlier, all the above is consistent with a need for both inner and outer scaling for the streamwise turbulence intensities in the near-wall region. The arguments would also apply to the spanwise turbulence intensities $\left(\overline{v^{2}} / U_{\tau}^{2}\right)$ but not to the wallnormal intensities $\left(\overline{w^{2}} / U_{\tau}^{2}\right)$ and the Reynolds shear stress $\left(-\overline{u w} / U_{\tau}^{2}\right)$ owing to the 


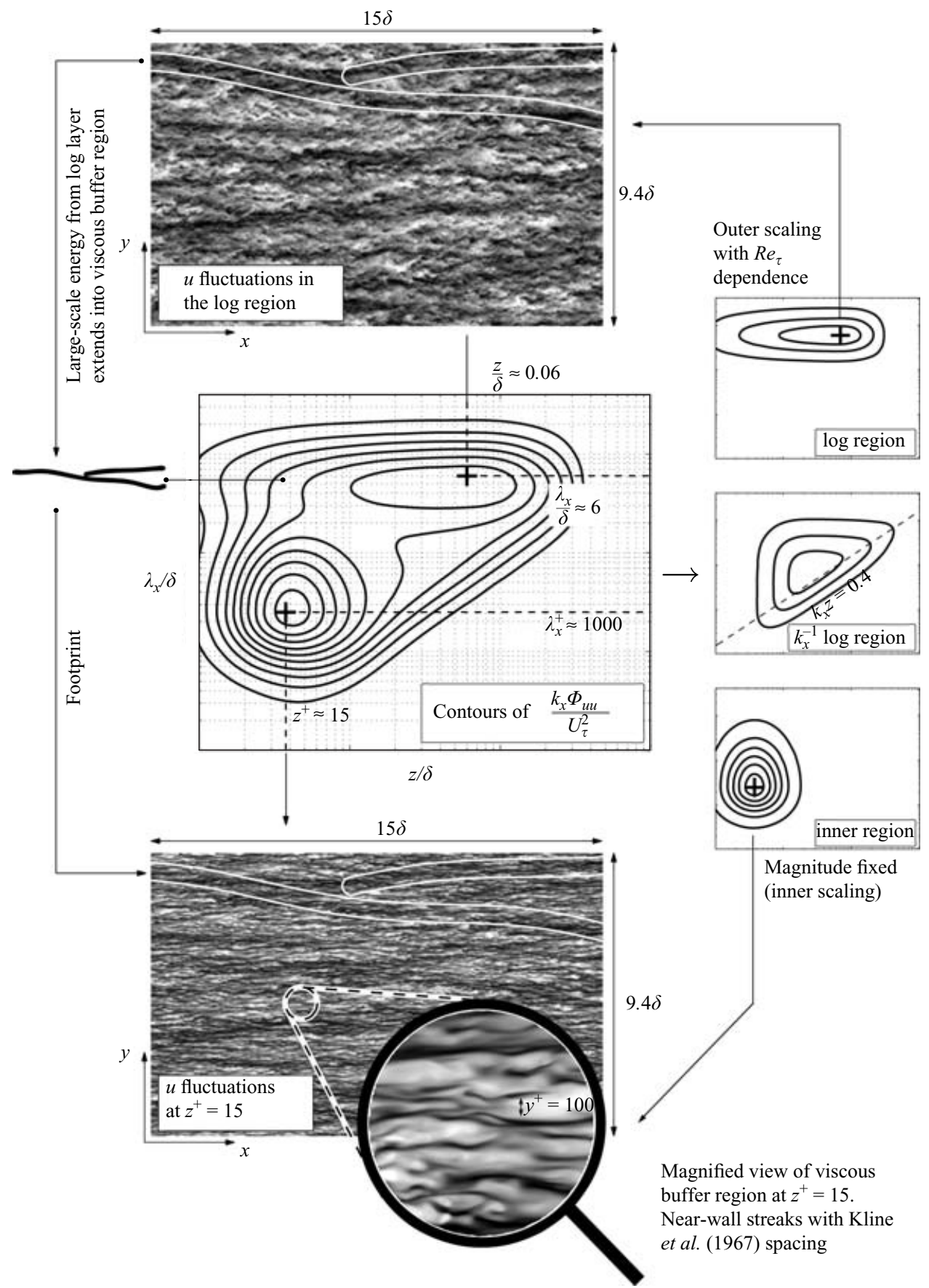

FIGURE 11. Schematic explaining the relative contributions to the energy spectra shown in figure 10. The main iso-contour plot has three salient components, as shown on the right-hand side.

blocking effect of the wall. (Within vortex-based models, one can also interpret this blocking effect as the image vortex structures in the wall.) Certainly this is reflected in experimental and DNS data by a spatial compactness of $w$ and Reynolds stress 
fluctuations as compared to $u$ and $v$ (Ganapathisubramani et al. 2003, 2005; Hoyas \& Jiménez 2006). However, an important fact that is often overlooked in these statistics is that although $w$ and $u w$ do not contain energy at low wavenumbers, the individual spatially compact concentrations exhibit a strong alignment with the extremely long $u$ modes of the superstructure (see Ganapathisubramani et al. 2003 and Marusic \& Hutchins 2006 for examples of this, and all literature pertaining to hairpin packet models).

Evidence of an outer-scale contribution to the near-wall region is not new, and has been noted in the literature for many decades. One particular study is that of Rao, Narasimha \& Badri Narayanan (1971), who considered scaling of the near-wall 'bursting' frequency. Using only single-point time-series data, they were able to deduce that large outer-scaled structures were active in rearranging and interacting with the near-wall structure. This is basically the same conclusion that we arrive at from our study.

\section{The atmospheric surface layer (ASL)}

Preliminary results from recent experiments performed in the atmospheric surface layer will also be briefly discussed. These data make a useful high Reynolds number appendix to the preceding discussion. Experiments were conducted at the SLTEST facility, located in Dugway Proving Grounds, Utah. Full details of the facility are available in Klewicki et al. (1995), Metzger \& Klewicki (2001) and Kunkel \& Marusic (2006). The unique geography of this site enables measurements to be made in extremely high Reynolds number turbulent boundary layers $\left(R e_{\tau} \approx O\left(10^{6}\right)\right)$ that have developed over $100 \mathrm{~km}$ of low surface roughness featureless salt flat (equivalent sand grain roughness $k^{+} \approx 22$ ). These atmospheric measurements are essentially a scaled-up version of the hot-wire rake experiments described in $\S 2$, with wall-normal position and spanwise separation scaled on the expected boundary layer thickness ( $\delta$ is estimated at $60 \mathrm{~m}$ for the ASL). A spanwise array of 10 sonic anemometers (Campbell Scientific CSAT3) are deployed on tripods mounted $2.14 \mathrm{~m}$ above the desert floor and spaced $3 \mathrm{~m}$ apart in the spanwise direction. At one end of the spanwise array is located an additional wall-normal array of 9 logarithmically spaced sonic anemometers (from $z=1.4$ to $25.7 \mathrm{~m}$ ). A photograph of the measurement array installed at the SLTEST site is included here as figure 12. Details of the experimental procedure will be reported in full elsewhere.

We will consider an hour of data taken from a period of neutral buoyancy and steady wind conditions during the early hours of 2 June 2005. Mean statistics from this hour compare well to canonical turbulent boundary layers measured in laboratory facilities. The mean velocity profile obtained from the wall-normal array is logarithmic, which together with $U_{\tau}$ indicates that the log-law extends from $9 \mathrm{~mm}$ above the wall to beyond the top of the tower (the entire tower shown in figure 12 is located within the log region). Turbulent intensities are as predicted by the similarity approximations of Marusic \& Kunkel (2003). Spanwise correlations of streamwise velocity fluctuation $\left(R_{u u}\right)$ for the spanwise array are shown in figure 13 and compare well to the correlations measured with the hot-wire rake (see $\S 4.1$ ). The symbols show the correlation data from laboratory measurements across the Reynolds number range $1840<R e_{\tau}<19960$. The solid lines show the corresponding data for the hour of ASL data. The collapse with outer scaling is remarkably good, especially considering that the comparison between the symbols and the lines represents a Reynolds number range of more than two decades (and $\delta$ has varied by three orders of magnitude). 


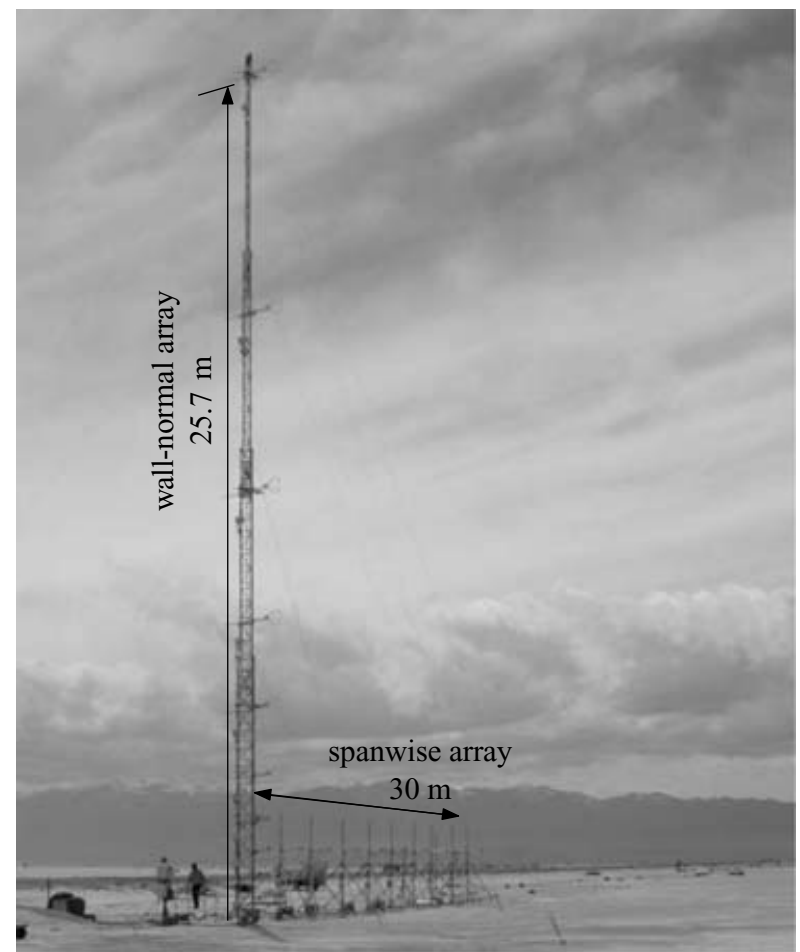

FIGURE 12. View of the measurement array installed at the SLTEST site.
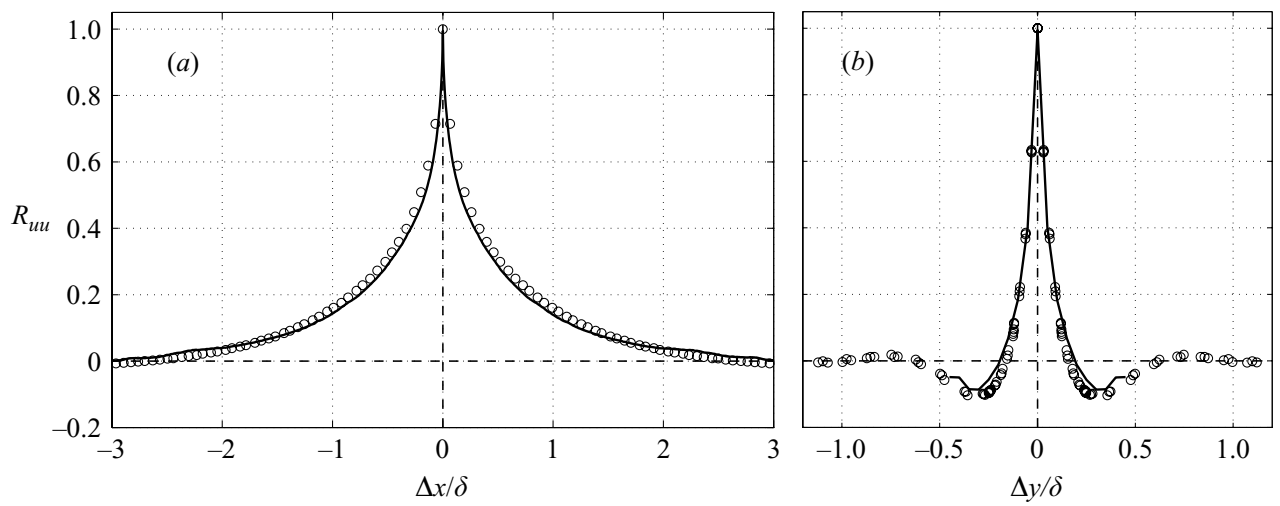

FIGURE 13. (a) Streamwise and $(b)$ spanwise two-point correlations of the streamwise velocity fluctuation $R_{\text {ии }}$ ( (O) show rake data for $1840<R e_{\tau}<19960$ at $z / \delta=0.05$; solid line shows Utah data for $R e_{\tau} \approx 660000$ at $z / \delta=0.036$.

There are some signs that the ASL correlations are not fully converged. Even with one hour of data, the total advection length for the ASL measurement is only approximately $300 \delta$ as compared to over $37000 \delta$ for the $R e_{\tau}=19960$ laboratory data. Convergence of low-wavenumber information will always be problematic in atmospheric measurements owing to limited periods of neutral stability and very large structural length scales. Regardless, figure 13 shows that similar large-scale features inhabit the log region of high Reynolds number atmospheric surface layers. The long region of positive correlation, flanked in the spanwise direction by anti-correlated 


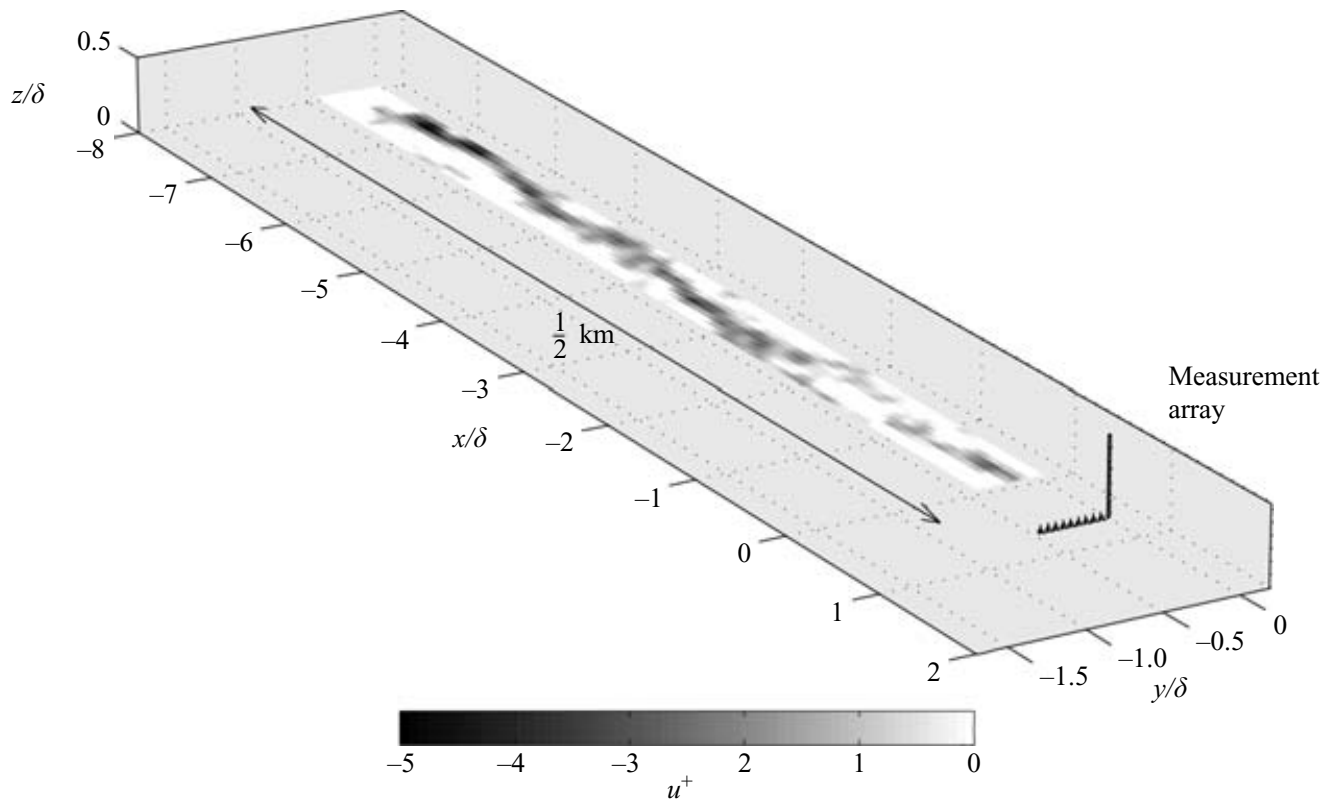

FIGURE 14. Example of signal across the spanwise array of sonic anemometers at SLTEST, $z / \delta=0.037, R e_{\tau}=660000$. The $x$-axis is reconstructed using Taylor's hypothesis and a convection velocity based on the local mean, $\bar{U}=5.46 \mathrm{~m} \mathrm{~s}^{-1}$. Shading shows only negative $u$ fluctuations (see grey scale).

behaviour is a typical statistical signature of the 'superstructure'. Figure 14 shows instantaneous streamwise velocity fluctuations for a $100 \mathrm{~s}$ trace across the spanwise array. The streamwise ordinate is reconstructed using Taylor's hypothesis, in exactly the same way as for figure 4. It is immediately obvious that the same very long meandering features inhabit the log region of the ASL. The feature shown in figure 14 is almost half a kilometre in length. Some sense of proportion can be obtained from the schematic of the measurement array, which is drawn to scale on the figure (compare to the photograph in figure 12). A comparison with laboratory results would indicate that even longer features will occur $(>20 \delta)$. Indeed, the meandering tendency of these large-scale features means that they often wander into and out of our measurement domain before we can assess their true length (the spanwise width of the sonic anemometer array is only $0.5 \delta$ and the sample length is also somewhat limited).

The feature shown in figure 4 is enormous in comparison to the near-wall structure (1000 wall units in the ASL equates to $90 \mathrm{~mm}$ ). With this kind of scale separation between the near-wall cycle and the log region structure, it becomes increasingly less intuitive to sanction a situation whereby the near-wall cycle can influence or give rise to the 'superstructure'. Certainly at lower Reynolds number the degree of scale-overlap tends to give the impression that these two scales are intimately entwined. Perhaps this is so at low Reynolds numbers, with the log region structure subject to a certain degree of wall-up interaction from the near-wall cycle. However, for the ASL, we are left with the notion that the inner and outer energy site (figure 11) could be two quite separate regimes, and that any substantial interaction is likely to be top-down (Hunt \& Morrison 2000). Circumstantial evidence for this scenario comes from Jiménez \& Pinelli (1999), who demonstrate that the near-wall cycle is autonomous and can 
continue largely unabated even in the absence of external (large-scale) influence. In addition, the existence of 'superstructure'-type features over fully roughened walls (where the near-wall cycle in theory no longer exists) implies that the $\log$ region structure is shear-driven rather than relying on a specific near-wall formation process.

\section{Conclusions}

The principle findings of this study are summarized by the following points.

(i) Superstructures. hot-wire rake measurements have confirmed that very long meandering features populate the log region of turbulent boundary layers, extending to over $20 \delta$ in length. These features form the stripiness noted in PIV experiments that has been previously related to the hairpin packet paradigm and shown to carry a large proportion of the Reynolds shear stress in the log region (Ganapathisubramani et al. 2003; Marusic \& Hutchins 2006). These may be what Kim \& Adrian (1999) referred to as very large-scale motions (VLSM) in turbulent pipe flow.

(ii) Outer scaling. the superstructures have a dimension that scales on boundary layer thickness $\delta$. Thus as Reynolds number increases they become increasingly large in comparison to the near-wall structure. Taking the inner and outer characteristic length scales from the local peaks in the pre-multiplied energy spectrum of $u$ (figure 10), we can approximate that the superstructure is larger than the nearwall structure by a factor of at least $6 R e_{\tau} / 1000$. The streamwise velocity fluctuations due to the superstructures appear to be Reynolds number-dependent. Further studies, including higher Reynolds number data, are likely required to fully determine their correct velocity scale.

(iii) Meandering. instantaneous views of $u$ fluctuation in the log region suggest that the superstructure meanders substantially along its length (see hot-wire rake, DNS and sonic anemometer data of figures 4, 7 and 14). By studying synthetic meandering flow features, it is shown that this meandering can severely curtail the length scales we infer from two-point correlations and pre-multiplied energy spectra $(\S 4.3)$. The fact that longer length scales are reported in the log region of pipe flows than for boundary layers (Kim \& Adrian 1999) may suggest that spanwise (or azimuthal) meandering is to some degree constrained in certain internal geometries.

(iv) Footprint. importantly, these very large outer-scaled structures maintain a footprint in the near-wall region. This is clear from instantaneous DNS data (figure 7) and the wall-normal variation of $k_{x} \Phi_{u u}$ (figure 10). This input of low-wavenumber outer-scaled energy into the near-wall region supports the need for both inner and outer scaling (and hence $R e_{\tau}$ dependency) of the near-wall streamwise turbulence intensities.

(v) Atmospheric surface layer. we have anchored our observations with a single high Reynolds number measurement made in the atmosphere at $R e_{\tau} \approx 660000$. For periods of steady wind and neutral buoyancy, we confirm the presence and scaling of superstructures in high-Reynolds number turbulent boundary layers $(\S 7)$.

(vi) Possible origins. numerous mechanisms have been suggested to explain the existence of large-scale features in the $\log$ and wake region of turbulent boundary layers, as discussed in $\S 1$. At this stage the authors lack the time-resolved information required to comment on formation mechanisms. However, our recent experiences in the ASL, where scale separation between the near-wall structure and superstructure is more than three orders of magnitude different, makes us somewhat wary of 'wallup'-type explanations. With increasing Reynolds number, the over-riding impression from our experiences at SLTEST and from the development of pronounced inner 
and outer sites in the energy spectra (figure 10) is that the inner- and outer-scaled structure could in fact be two quite separate regimes that overlap and are intertwined in some complex Reynolds number-dependent manner.

The preceding analysis has dealt exclusively with streamwise velocity fluctuations. In future work we would like to expand the measurements made here to include the remaining two velocity components. Of particular interest are the pre-multiplied spectra for the spanwise fluctuations $\left(k_{x} \Phi_{v v}\right)$, which are predicted to scale in a similar manner to the $u$ spectra. This is also the component for which the least amount of experimental data presently exists.

The authors gratefully acknowledge support from the National Science Foundation (Grant CTS-0324898) and the David and Lucile Packard Foundation. The authors also wish to thank R. D. Moser for kindly making the $R e_{\tau}=934$ DNS database available, M. S. Chong for hosting the Melbourne experiments, and William Hambleton and Todd Novak for assisting with some of the experiments. The SLTEST measurements were a collaborative effort involving a large team from several universities. Specifically, we acknowledge Joe Klewicki of the University of Utah, and Keith McNaughton of the University of Edinburgh.

\section{Appendix A. Functional form of synthetic flow fields}

In $\S 4.3$, streamwise-aligned and spanwise-meandering synthetic streak arrays were considered (cases I and II respectively). The spanwise profile of case I is invariant in $x$, and given by

$$
\begin{aligned}
& \text { case I: } \quad-\frac{3 L_{y}}{2}<y_{f I}<\frac{3 L_{y}}{2}, \quad 0<x_{f I}<L_{x} \\
& u_{f I}\left(y_{f I}, x_{f I}\right)=\left(-\frac{3}{4}-\frac{\cos \left(\pi y_{f I} / L_{y}\right)}{4 \sqrt{\cos ^{2}\left(\pi y_{f I} / L_{y}\right)}}\right) \cos \left(\frac{\pi y_{f I}}{L_{y}}\right), \\
& \begin{array}{c}
\text { Spanwise profile } \\
\text { of fake streak }
\end{array}
\end{aligned}
$$

where $L_{y}$ is the streak width and $L_{x}$ is the streak length. The accompanying sketch shows that this function describes $1 \frac{1}{2}$ periods of a co-sinusoid modulated by a box function such that the integral of the signal in the spanwise direction is zero. For the spanwise meandering features of case II, the original function $\left(u_{f I I}\right)$ has an $x$-dependent sinusoidal shift applied in the spanwise direction, of the form

$$
u_{f I I}\left(y_{f I I}, x_{f I I}\right)=u_{f I}
$$

where

$$
y_{f I I}=A \sin \left(2 \pi x_{f} / \omega+\phi\right)+y_{f I} .
$$


(a)

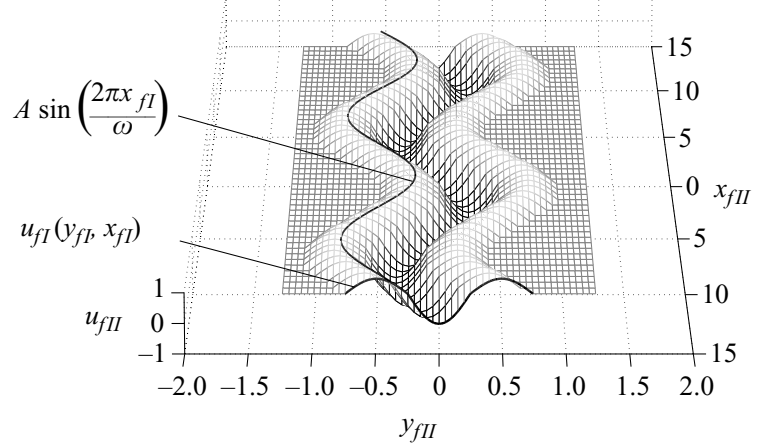

(b)

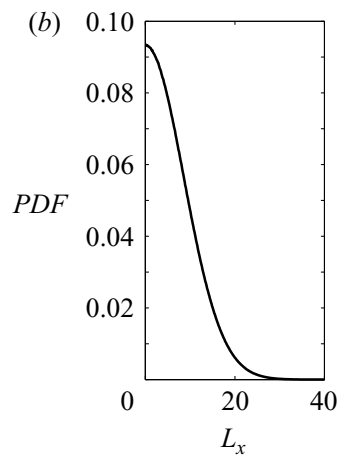

FIgURE 15. (a) Case II: the meandering fake streak, as given by (A 2); (b) length scale distribution for $L_{x}$.

$A$ and $\omega$ are the respective amplitude and period of the meander and $\phi$ (which is uniformly distributed between 0 and $\omega$ ) randomizes the phase of the meander in $x_{f}$. A sketch of the meandering case is given in figure $15(a)$.

To create the full synthetic flow fields, the aligned or meandering features are randomly arranged with a normal distribution of length scales (about zero), yielding a mean length of $\bar{L}_{x} \approx 7 \delta$ and a maximum length of approximately $30 \delta$. Figure $15(b)$ shows the normalized probability distribution of $L_{x}$.

\section{Appendix B. A note on energy spectra}

The analysis of $\S 4.3$ shows that meandering may lead to misinference of length scales from one-dimensional energy spectra. In fact, there is potential for misinterpretation even when no meandering is present. Consider the pre-multiplied energy spectra for the streamwise-aligned case as shown by the solid line of figure $5(d)$. The peak in this curve occurs at $18 \delta$, despite the fact that the average structural length for this case was just $6.9 \delta$ (as given by the distribution in figure $15 b$ ). Two important factors contribute to this discrepancy. In the first instance, the structure that we have modelled represents only approximately one half of a sinusoidal period in the streamwise direction. In such cases we would expect a spectral peak to occur at roughly twice the characteristic length scale. The actual streamwise profile used for the synthetic flows is a Hanning window of length $L_{x}$, which can be shown to yield a spectral peak at $1.717 L_{x}$ (obtained by regression fit to a sinusoid). Thus it is clear that where flows are non-sinusoidally periodic (as turbulence indeed is) it is perhaps unwise to rely purely on a sinusoidal decomposition to infer structure. This factor alone does not fully account for the discrepancy in figure $5(d)$. There is an additional problem due to length scale distribution. Campbell's theorem tells us that randomly arranged events of equal length will return an energy spectrum identical in shape to that of a single isolated event. In this sense we might hope to be able to back-out structural information from energy spectra. However, in the presence of any form of distribution of length scales, Campbell's theorem informs us that the final energy spectrum will be a summation of the spectra for each individual length scale modulated by a density factor (probability $\times$ length scale). Thus the final spectral estimate (including the location of any pre-multiplied peak) is contaminated by the length scale distribution. The wide distribution of overlapping scales makes turbulent signals a prime candidate for this form of contamination. 

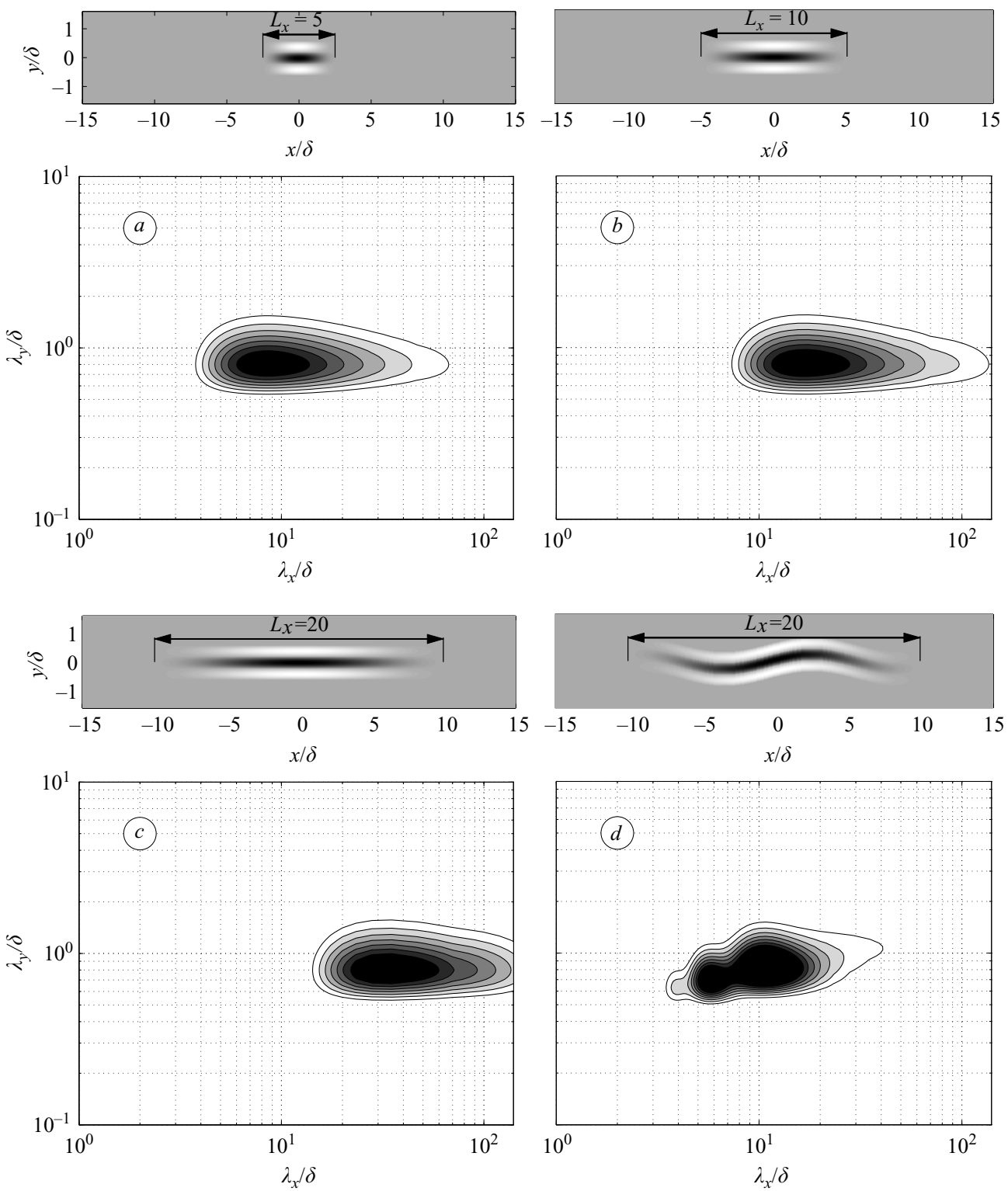

FIGURE 16. Two-dimensional premultiplied energy spectra for various isolated synthetic flow fields. Filled contours show normalized magnitude of $k_{x} k_{y} E_{u u}$ for $(a)$ case I, streamwise-aligned $L_{x}=5 \delta ;(b)$ case I, streamwise-aligned $L=10 \delta ;(c)$ case I, streamwise-aligned $L=20 \delta$; (d) case II, meandering $L=20 \delta$. In each case a schematic of the synthetic flow is shown above the spectra (where the grey scale shows streamwise velocity fluctuation).

Two-dimensional spectra $\left(E_{u u}\right)$ are not immune to these problems. Figure 16 shows $k_{x} k_{y} E_{u u}$ for various synthetic flow functions. Plots $(a)$ to $(c)$ show streamwise-aligned cases. For these single events, the peak in $\lambda_{x}$ occurs at $1.717 L_{x}$, as was previously predicted for the Hanning function. In the spanwise direction the peak in $\lambda_{y}$ occurs at $2 L_{y}$ (since the spanwise waveform is essentially sinusoidal). It is noted that in all cases the low wavenumber energy in the streamwise direction persists to approximately 10 
times the peak value in $\lambda_{x}$. When analysing turbulent flow data it would be easy to misconstrue this tail in the low wavenumber energy as indicating the presence of very long structures. Yet for the synthetic flow fields under investigation there are no features with lengths greater than $L_{x}$. Figure $16(d)$ shows the $L_{x}=20 \delta$ case with meandering. The comparison between plots $(c)$ and $(d)$ highlights once again how the addition of meandering can shift the peak in the premultiplied spectra to shorter wavelengths. Not only this, but the meandering has also inclined the contours of $k_{x} k_{y} E_{\text {uи }}$ in the $k_{y}$ direction. It is noted that contours of $k_{x} k_{y} E_{\text {uи }}$ calculated from DNS channel flows are tilted in this manner (del Álamo et al. 2004). It would be tempting to assume from the inclined contours of figure $16(d)$ that structures increase in width as they become longer. However, for the synthetic input structure used here, we know that this is not really the case. Rather, it is true that as structures become longer, they are statistically more likely to wander over a wider spanwise domain.

\section{REFERENCES}

Abe, H., Kawamura, H. \& CHOI, H. 2004 Very large-scale structures and their effects on the wall shear-stress fluctuations in a turbulent channel flow up to $R e_{\tau}=640$. Trans. ASME: J. Fluids Engng 126, 835-843.

Adrian, R. J., Meinhart, C. D. \& Tomkins, C. D. 2000 Vortex organization in the outer region of the turbulent boundary layer. J. Fluid Mech. 422, 1-54.

Del Álamo, J. C. \& JimÉnez, J. 2003 Spectra of the very large anisotropic scales in turbulent channels. Phys. Fluids 15, 41-44.

del Álamo, J. C., Jiménez, J., Zandonade, P. \& Moser, R. D. 2004 Scaling of the energy spectra of turbulent channels. J. Fluid Mech. 500, 135-144.

Balint, J.-L., Wallace, J. M. \& Vukoslavcevic, P. 1991 The velocity and vorticity vector fields of a turbulent boundary layer. Part 2. Statistical properties. J. Fluid Mech. 228, 53-86.

Blackwelder, R. F. \& Kovasznay, L. S. G. 1972 Time scales and correlations in a turbulent boundary layer. Phys. Fluids 15, 1545-1554.

Ching, C., Duenidi, L. \& Antonia, R. 1995 Low-Reynolds-effects in a turbulent boundary layer. Exps. Fluids 19, 61-68.

DeGraAfF, D. B. \& Eaton, J. K. 2000 Reynolds number scaling of the flat-plate turbulent boundary layer. J. Fluid Mech. 422, 319-346.

Drobinski, P., Carlotti, P., Newsom, R. K., Banta, R. M., Foster, R. C. \& Redelsperger, J.-L. 2004 The structure of the near-neutral atmospheric surface layer. J. Atmos. Sci. 61, 699-714.

Ganapathisubramani, B., Hutchins, N., Hambleton, W. T., Longmire, E. K. \& Marusic, I. 2005 Investigation of large-scale coherence in a turbulent boundary layer using two-point correlations. J. Fluid Mech. 524, 57-80.

Ganapathisubramani, B., Longmire, E. K. \& Marusic, I. 2003 Characteristics of vortex packets in turbulent boundary layers. J. Fluid Mech. 478, 35-46.

Guala, M., Hommema, S. E. \& Adrian, R. J. 2006 Large-scale and very-large-scale motions in turbulent pipe flow. J. Fluid Mech. 554, 521-542.

Hafez, S., Chong, M. S., Marusic, I. \& Jones, M. B. 2004 Observations on high Reynolds number turbulent boundary layer measurements. In Proc. 15th Australasian Fluid Mech. Conf (ed. M. Behnia, W. Lin \& G. D. McBain), Paper AFMC 00200. University of Sydney.

Hambleton, W. T., Hutchins, N. \& Marusic, I. 2006 Multiple plane PIV measurements in a turbulent boundary layer. J. Fluid Mech. 560, 53-64, in press.

Hoyas, S. \& JimÉnEZ, J. 2006 Scaling of the velocity fluctuations in turbulent channels up to $R e_{\tau}=2003$. Phys. Fluids 18, 011702 .

Hunt, J. C. R. \& Morrison, J. F. 2000 Eddy structure in turbulent boundary layers. Eur. J. Mech. B-Fluids 19, 673-694.

Hutchins, N., Ganapathisubramani, B. \& Marusic, I. 2004 Dominant spanwise Fourier modes, and the existence of very large scale coherence in turbulent boundary layers. In Proc. 15th 
Australasian Fluid Mech. Conf. (ed. M. Behnia, W. Lin \& G. D. McBain), Paper AFMC 00127. University of Sydney.

Hutchins, N., Ganapathisubramani, B. \& Marusic, I. $2005 a$ Spanwise periodicity and the existence of very large scale coherence in turbulent boundary layers. In Proc. Fourth Intl Symposium on Turbulence and Shear Flow Phenomena, pp. 39-44. (TSFP4, Willamsburg, Virginia).

Hutchins, N., Hambleton, W. T. \& Marusic, I. $2005 b$ Inclined cross-stream stereo particle image velocimetry measurements in turbulent boundary layers. J. Fluid Mech. 541, 21-54.

Iwamoto, K., Suzuki, Y. \& Kasagi, N. 2002 Reynolds number effect on wall turbulence: Toward effective feedback control. Intl J. Heat Fluid Flow 23, 678-689.

JimÉnEZ, J. 1998 The largest scales of turbulent wall flows. In CTR Annual Research Briefs, pp. 943-945. Stanford University.

JimÉnez, J. \& DEL Álamo, J. C. 2004 Computing turbulent channels at experimental Reynolds numbers. In Proc. 15th Australasian Fluid Mech. Conf. (ed. M. Behnia, W. Lin \& G. D. McBain), Paper AFMC 00038. University of Sydney.

JimÉnez, J., Del Álamo, J. C. \& Flores, O. 2004 The large-scale dynamics of near-wall turbulence. J. Fluid Mech. 505, 179-199.

JimÉnez, J. \& Pinelli, A. 1999 The autonomous cycle of near-wall turbulence. J. Fluid Mech. 389, 335-359.

Johansson, A., Her, J.-Y. \& Haritonidis, J. 1987 On the generation of high-amplitude wall-pressure peaks in turbulent boundary layers and spots. J. Fluid Mech. 175, 119-142.

Johansson, T. \& KarLsSON, R. 1989 The energy budget in the near-wall region of a turbulent boundary layer. In Applications of Laser Anemometry to Fluid Mechanics (ed. R. Adrian, T. Asanuma, D. Durao, F. Durst \& J. Whitelaw), pp. 3-22. Springer.

Kim, K. C. \& Adrian, R. 1999 Very large-scale motion in the outer layer. Phys. Fluids 11, 417-422.

KLEWICKI, J. C. \& FALCO, R. E. 1990 On accurately measuring statistics associated with small scales in turbulent boundary layers using hot-wire probes. J. Fluid Mech. 219, 119-142.

Klewicki, J. C., Metzger, M. M., Kelner, E. \& Thurlow, E. M. 1995 Viscous sublayer flow visualizations at $R_{\theta} \cong 1500000$. Phys. Fluids 7, 857-863.

Kline, S. J., Reynolds, W. C., Schraub, F. A. \& Rundstadler, P. W. 1967 The structure of turbulent boundary layers. J. Fluid Mech. 30, 741-773.

Kovasznay, L. S. G. 1970 The turbulent boundary layer. Annu. Rev. Fluid Mech. 2, 95-112.

Kovasznay, L. S. G., Kibens, V. \& Blackwelder, R. F. 1970 Large-scale motion in the intermittent region of a turbulent boundary layer. J. Fluid Mech. 41, 283-326.

Kunkel, G. J. \& Marusic, I. 2006 Study of the near-wall-turbulent region of the high-Reynoldsnumber boundary layer using an atmospheric flow. J. Fluid Mech. 548, 375-402.

Ligrani, P. M. \& Bradshaw, P. 1987 Spatial resolution and measurement of turbulence in the viscous sublayer using subminiature hot-wire probes. Exps. Fluids 5, 407-417.

Marusic, I. 2001 On the role of large-scale structures in wall turbulence. Phys. Fluids 13, 735-743.

Marusic, I. \& Hutchins, N. 2006 Experimental study of wall turbulence: Implications for control. In Transition and Turbulence Control (ed. M. Gad-el-Hak \& H. M. Tsai). World Scientific.

Marusic, I. \& Kunkel, G. J. 2003 Streamwise turbulence intensity formulation for flat-plate boundary layers. Phys. Fluids 15, 2461-2464.

Marusic, I. \& PerRY, A. E. 1995 A wall wake model for the turbulent structure of boundary layers. Part 2. Further experimental support. J. Fluid Mech. 298, 389-407.

MCLean, I. R. 1990 The near-wall eddy structure in an equilibrium turbulent boundary layer. $\mathrm{PhD}$ thesis, University of Southern California, USA.

Metzger, M. M. \& KLewicki, J. C. 2001 A comparative study of near-wall turbulence in high and low Reynolds number boundary layers. Phys. Fluids 13, pp. 692-701.

Metzger, M. M., Klewicki, J. C., Bradshaw, K. L. \& Sadr, R. 2001 Scaling the near-wall axial turbulent stress in the zero pressure gradient boundary layer. Phys. Fluids 13, 1819-1821.

Moser, R. D., Kim, J. \& Mansour, N. N. 1999 Direct numerical simulation of turbulent channel flow up to $R e_{\tau}=590$. Phys. Fluids 11, 943-945.

Nakagawa, H. \& NeZU, I. 1981 Structure of space-time correlations of bursting phenomena in an open-channel flow. J. Fluid Mech. 104, 1-43.

Nickels, T. B., Marusic, I., Hafez, S. \& Chong, M. S. 2005 Evidence of the $k_{1}^{-1}$ law in a high-Reynolds-number turbulent boundary layer. Phys. Rev. Lett. 95, 074501. 
Perry, A. E., Henbest, S. \& Chong, M. S. 1986 A theoretical and experimental study of wall turbulence. J. Fluid Mech. 165, 163-199.

Perry, A. E. \& Marusic, I. 1995 A wall wake model for the turbulent structure of boundary layers. Part 1. Extension of the attached eddy hypothesis. J. Fluid Mech. 298, 361-388.

Phillips, W. R. C. 2003 Langmuir circulations. In Wind over Waves II: Forecasting and Fundamentals of Applications (ed. S. G. Sajjadi \& J. C. R. Hunt), pp. 157-167. Horwood.

Purtell, P., Klebanoff, P. \& Buckley, F. 1981 Turbulent boundary layer at low Reynolds number. Phys. Fluids 24, 802-811.

RaO, K. N., NARAsimha, R. \& Badri Narayanan, M. A. 1971 The 'bursting' phenomena in a turbulent boundary layer. J. Fluid Mech. 48, 339-352.

Schoppa, W. \& Hussain, F. 2002 Coherent structure generation in near-wall turbulence. J. Fluid Mech. 453, 57-108.

SpAlaRT, P. R. 1988 Direct numerical simulation of a turbulent boundary layer upto $R_{\theta}=1410$. J. Fluid Mech. 187, 61-98.

Tanahashi, M., Kang, S.-J., Miyamoto, T., Shiokawa, S. \& Miyauchi, T. 2004 Scaling law of fine scale eddies in turbulent channel flows up to $R e_{\tau}=800$. Intl J. Heat Fluid Flow 25, 331-340.

TOH, S. \& ITANO, T. 2005 Interaction between a large-scale structure and near-wall structures in channel flow. J. Fluid Mech. 524, 249-262.

Tomkins, C. D. \& Adrian, R. J. 2003 Spanwise structure and scale growth in turbulent boundary layers. J. Fluid Mech. 490, 37-74.

Townsend, A. A. 1956 The Structure of Turbulent Shear Flow. Cambridge University Press.

TsubokurA, M. 2005 LES study on the large-scale motions of wall turbulence and their structural difference between plane channel and pipe flows. In Proc. Fourth Intl Symposium on Turbulence and Shear Flow Phenomena, pp. 1037-1042. TSFP4, Willamsburg, Virginia.

UedA, H. \& Hinze, J. O. 1975 Fine-structure turbulence in the wall region of a turbulent boundary layer. J. Fluid Mech. 67, 125-143.

Wark, C. E., Naguib, A. M. \& Robinson, S. K. 1991 Scaling of spanwise length scales in a turbulent boundary layer. AIAA Paper 91-0235.

Wei, T. \& WillmaRTH, W. W. 1989 Reynolds-number effects on the structure of a turbulent channel flow. J. Fluid Mech. 204, 57-95.

Young, G. S., Kristovich, D. A. R., Huelmfelt, M. R. \& Foster, R. C. 2002 Rolls, streets, waves and more: A review of quasi-two-dimensional structures in the atmospheric boundary layer. Bull: Am. Met. Soc. 83, 9971001. 


\section{University Library}

\section{- M M I N E R VA A gateway to Melbourne's research publications}

Minerva Access is the Institutional Repository of The University of Melbourne

Author/s:

Hutchins, N.;MARUSIC, IVAN

Title:

Evidence of very long meandering features in the logarithmic region of turbulent boundary layers

Date:

2007

Citation:

Hutchins, N., \& Marusic, I. (2007). Evidence of very long meandering features in the logarithmic region of turbulent boundary layers. Journal of Fluid Mechanics, 579, 1-28.

Publication Status:

Published

Persistent Link:

http://hdl.handle.net/11343/33549 